\begin{tabular}{|c|c|c|}
\hline Beitr. Ent. & Keltern & ISSN 0005-805X \\
\hline $\mathbf{5 9}(2009) 1$ & S. $133-173$ & 15.07 .2009 \\
\hline
\end{tabular}

\title{
Nuovi dati faunistici e tassonomici su Aleocharinae delle tribù Lomechusini, Hoplandriini, Oxypodini e Aleocharini del Sudamerica
}

\section{(Coleoptera, Staphylinidae)}

\author{
Con 113 figure
}

Roberto Pace

\section{Summary}

Taxonomic and faunal data of the 132 specimens in the Zoological Museum of the Humboldt University, Berlin, the Deutsches Entomologisches Institut, Müncheberg and the Herbert Franz Collection in the Naturhistorisches Museum, Wien, are provided. Four tribes (Lomechusini, Hoplandriini, Oxypodini and Aleocharini), 19 genera (Orphnebius, Apalonia, Myrmecoxenia, Macrogerodonia, Zyras, Abrophaena, Meoticaops, Apimela, Feluva, Idiostiba, Diacanthochara, Acrocyusa, Neocalodera, Spanioda, Tinotus, Platandria, Hoplandria, Ctenopeuca and Aleochara) and 50 species are recognized. Thirty-seven species new to science are described and illustrated. The new species are compared with similar species. Three new combinations are proposed.

\section{Key words}

Coleoptera, Staphylinidae, Aleocharinae, taxonomy, South America.

\section{Zusammenfassung}

Es werden taxonomische und faunistische Angaben von 132 Aleocharinen aus dem Zoologischen Museum der Humboldt Universität zu Berlin, aus dem Deutschen Entomologischen Institut in Müncheberg und aus der Sammlung Herbert Franz aus dem Naturhistorischen Museum in Wien dargestellt. Dabei können 50 Arten in 4 Tribus (Lomechusini, Hoplandriini, Oxypodini und Aleocharini) und 19 Gattungen (Orphnebius, Apalonia, Myrmecoxenia, Macrogerodonia, Zyras, Abrophaena, Meoticaops, Apimela, Feluva, Idiostiba, Diacanthochara, Acrocyusa, Neocalodera, Spanioda, Tinotus, Platandria, Hoplandria, Ctenopeuca und Aleochara) determiniert werden. Weiterhin werden 37 Arten neu beschrieben, mit ähnlicher Art verglichen und illustriert. Schließlich werden 3 neue Kombinationen vorgeschlagen.

\section{Riassunto}

Sono forniti dati tassonomici e faunistici di 132 esemplari del Museo Zoologico dell'Università Humboldt di Berlino, del Deutsches Entomologisches Institut di Müncheberg e della collezione di Herbert Franz al Naturhistorisches Museum di Vienna. Sono riconosciuti 4 tribù (Lomechusini, Hoplandriini, Oxypodini e Aleocharini), 19 generi (Orphnebius, Apalonia, Myrmecoxenia, Macrogerodonia, Zyras, Abrophaena, Meoticaops, Apimela, Feluva, Idiostiba, Diacanthochara, Acrocyusa, Neocalodera, Spanioda, Tinotus, Platandria, Hoplandria, Ctenopeuca e Aleochara) e 50 specie. Sono descritte e illustrate come nuove per la scienza 37 specie. Le nuove specie sono confrontate con specie affini. Sono proposte tre nuove combinazioni.

${ }^{1} 223^{\circ}$ Contributo alla conoscenza delle Aleocharinae. 


\section{Introduzione}

Le quattro tribù di Aleocharinae qui trattate comprendono specie di difficile attribuzione generica. Particolarmente le specie della tribù Lomechusini hanno qui attribuzione generica di carattere provvisorio. Grazie all'esame dei caratteri dell'organo copulatore maschile, dei segmenti genitali maschili e femminili e della spermateca, tuttavia sono sufficientemente ben riconoscibili al fine di futuri approfondimenti. Prima della pubblicazione di alcuni miei lavori sulle Aleocharinae del Sudamerica, nessun lavoro era stato pubblicato con disegni di habitus, edeago e spermateca che sono di indispensabile aiuto nella determinazione di esemplari. Il materiale di studio come quello disponibile per il presente lavoro, è troppo scarso per affrontare revisioni a livello generico e specifico, opportune in presenza di numerose specie descritte in modo frammentario. È ovvio che le specie qui rese note rappresentano una minima parte della diversità realmente esistente in natura.

\section{Materiale e Metodo}

Gli esemplari del presente lavoro appartengono alle collezioni del Museo Zoologico dell'Università Humboldt di Berlino, del DEI di Müncheberg, e del compianto prof. Herbert Franz, ora conservate al Naturhistorisches Museum di Vienna.

Gli esemplari sono stati dissezionati con lo scopo di includere le strutture genitali in balsamo del Canadà (su piccoli rettangoli trasparenti di materiale di plastica, che accompagnano gli esemplari). Le strutture genitali sono state studiate con l'uso di un microscopio composto fino a 450 ingrandimenti e disegnate per mezzo di oculare a reticolo. L'habitus è stato da me disegnato con l'ausilio di un oculare con scala micrometrica. Tutti i disegni delle tavole sono stati da me eseguiti fino alla fase finale. Le tavole sono state da me composte al computer.

Acronimi

DEI Deutsches Entomologisches Institut di Müncheberg

MB Museo di Storia Naturale dell'Università Humboldt di Berlino

NHMW Naturhistorisches Museum di Vienna

MRSNT Museo regionale di Scienze naturali di Torino

\section{Elenco delle specie note e descrizione dei nuovi taxa}

\section{Lomechusini}

Orphnebius maracayensis n. sp.

(Figg. 1-2)

Materiale tipico: Holotypus + , Venezuela, Rancho Grande, b. Maracay, leg. H. Franz (NHMW).

\section{Descrizione:}

Lunghezza 1,7 mm. Corpo lucido e bruno-rossiccio con pronoto e pigidio rossicci; antenne brune con i tre antennomeri basali gialli; zampe giallo-rossicce con femori giallo-bruni. Granulosità del capo, del pronoto e delle elitre saliente. Corpo senza reticolazione. Ciascuna elitra con una fossetta laterale. Uroterghi liberi nudi, privi di pubescenza. Spermateca fig. 2. 


\section{Comparazioni:}

L'habitus della nuova specie è simile a quello di O. lativentris SHARP, 1883, del Guatemala. Se ne distingue per il colore delle antenne bruno fino all'apice (antenne gialle all'estremità apicale in lativentris), per il penultimo antennomero lungo quanto largo (fortemente trasverso in lativentris) e per le elitre granulose (senza granuli e punteggiatura in lativentris).

Etimologia: La nuova specie prende nome dalla città di Maracay.
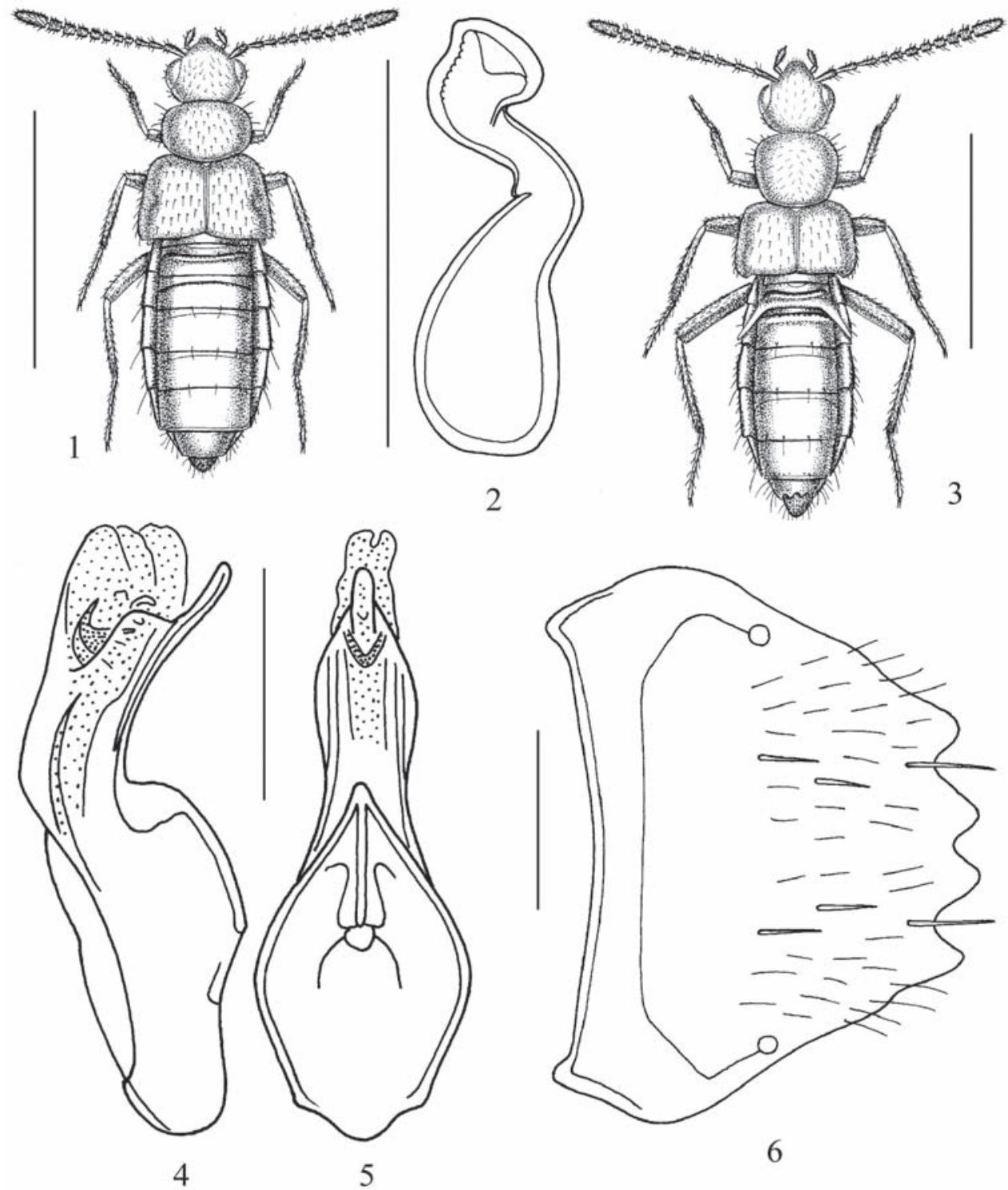

6

Figg. 1-6: Habitus, spermateca, edeago in visione laterale e ventrale e sesto urotergo libero del maschio. 1-2. Orphnebius maracayensis n. sp.; 3-6. Orphnebius satan n. sp. Habitus scala $1 \mathrm{~mm}$, altre scale 0,1 mm. 


\section{Orphnebius satan n. sp.}

(Figg. 3-6)

Materiale tipico: Holotypus ơ, Peru, Umg. Tarapoto, leg. H. Franz (NHMW).

\section{Descrizione:}

Lunghezza 2,1 mm. Corpo lucido e bruno-rossiccio; antenne bruno-rossicce con apice distale dell'undicesimo antennomero giallo-bruno; zampe gialle con femori rossicci. Punteggiatura visibile solo sulle tempie, quella del pronoto assente. Granulosità delle elitre molto superficiale. Corpo senza reticolazione. Primo urotergo libero del maschio prolungato in due spine divergenti all'indietro. Edeago figg. 4-5, sesto urotergo libero del maschio fig. 6.

\section{Comparazioni:}

La nuova specie è differente da O. pungitius Solsky, 1875, pure del Perù, per il pronoto poco trasverso e senza impressioni anteriori (pronoto molto trasverso e con solchi o impressioni tortuose in pungitius) e per i caratteri sessuali secondari dell'addome completamente differenti (vedi in Solsky, 1875 tavola I, fig. 2).

Etimologia: La nuova specie prende nome dal demonio «Satana» a motivo della forca a due denti del primo urotergo libero del maschio.

\section{Orphnebius amazonicus n. sp.}

(Figg. 7-9)

Materiale tipico: Holotypus क, Brasil, Amazonasgebiet, Umg. Manaus, leg. I. Beck (NHMW).

\section{Descrizione:}

Lunghezza 2,1 mm. Corpo lucido e bruno-rossiccio con elitre brune a base bruno-rossiccia; antenne bruno-rossicce con i due antennomeri basali e l'undicesimo rossicci; zampe anteriori e posteriori rossicce, zampe medie rossicce con tibie brune tranne che alle estremità. Punteggiatura del capo e del pronoto assente. Granulosità delle elitre fine e poco saliente. Corpo senza reticolazione. Uroterghi liberi nudi, senza pubescenza. Spermateca fig. 8.

\section{Comparazioni:}

La nuova specie è nettamente differente da O. tuberculatus Bernhauer, 1908, pure del Brasile, per le elitre non coperte di forti granuli come in tuberculatus.

Etimologia: La nuova specie prende nome dal territorio dell'Amazzonia.

\section{Orphnebius belemensis n. sp.}

(Figg. 10-13)

Materiale tipico: Holotypus ơ, Brasilien, Umg. Belem Para, leg. H. Franz (NHMW). Paratypus: 1 đَ, Brasilien, Umg Capitao Poco, Juni 1983, leg. H. Franz (MRSNT). 


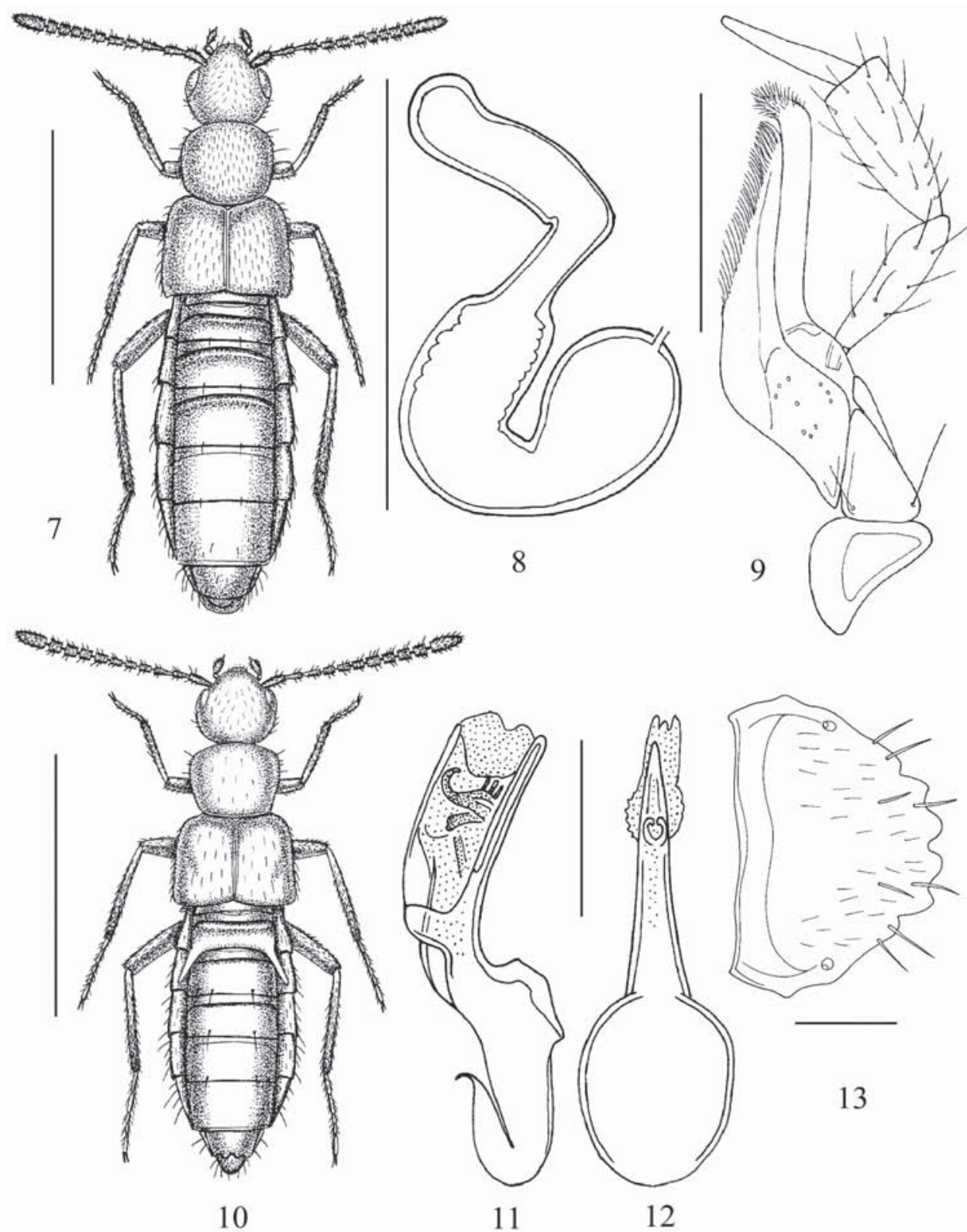

Figg. 7-13: Habitus, spermateca, maxilla con palpo mascellare, edeago in visione laterale e ventrale e sesto urotergo libero del maschio. 7-9. Orphnebius amazonicus n. sp.; 10-13. Orphnebius belemensis n. sp. Habitus scala $1 \mathrm{~mm}$, altre scale $0,1 \mathrm{~mm}$.

\section{Descrizione:}

Lunghezza 2,1 mm. Corpo lucido e bruno-rossiccio con i due uroterghi liberi basali rossicci; antenne bruno-rossicce con antennomero basale giallo-bruno e undicesimo giallo; zampe gialle con femori bruni. Punteggiatura del capo invisibile. Granulosità delle elitre rada e superficiale. Uroterghi liberi nudi, senza pubescenza. Edeago figg. 11-12, sesto urotergo libero del maschio fig. 13. 


\section{Comparazioni:}

La nuova specie è nettamente differente da O. tuberculatus Bernhauer, 1908, pure del Brasile, per le elitre non coperte di forti granuli come in tuberculatus.

Etimologia: La nuova specie prende nome dalla città di Belem.

\section{Orphnebius caligatus n. sp.}

(Figg. 14-15)

Materiale tipico: Holotypus ㅇ, Brasilien, Nova Teutonia, VI.1960, leg. F. Plaumann (MB).

\section{Descrizione:}

Lunghezza 3,4 mm. Corpo assai lucido e bruno con elitre bruno-rossicce; antenne brune con antennomero basale bruno-rossiccio e secondo e terzo basali giallo-rossicci; femori bruni, tibie e tarsi anteriori giallo-rossicci, tibie medie e posteriori bruno-rossicce con le due estremità e tarsi giallo-rossicci. Punteggiatura del capo e del pronoto molto superficiale. Granulosità delle elitre saliente. Uroterghi liberi nudi, senza pubescenza. Due punti isolati sul pronoto. Quinto urotergo libero della femmina con due forti granuli posteriori lungo il margine, sesto urotergo libero della femmina pure con due forti granuli su una superficie fortemente punteggiata. Spermateca fig. 15 .

\section{Comparazioni:}

La nuova specie è differente da O. tuberculatus Bernhauer, 1908, pure del Brasile, per il capo nettamente più stretto del pronoto che non presenta alcuni punti tra gli occhi (capo poco più stretto del pronoto e con punti tra gli occhi in tuberculatus) e manca una bozza del pronoto davanti allo scutello come in tuberculatus.

Etimologia: La nuova specie per le zampe posteriori di due colori sembra calzare le calighe, calzature dell'antica Roma.

\section{Orphnebius modestus n. sp.}

(Figg. 16-19)

Materiale tipico: Holotypus $0^{\star}$, Brasilien, Nova Teutonia, VI.1960, leg. F. Plaumann (MB).

Paratypus: 1 ㅇ, stessa provenienza (MRSNT).

\section{Descrizione:}

Lunghezza $2 \mathrm{~mm}$. Corpo lucidissimo e bruno-rossiccio con addome bruno; antenne brune con i tre antennomeri basali gialli; zampe gialle. Punteggiatura del capo e del pronoto assente. Corpo senza reticolazione. Granulosità delle elitre fine e saliente. Uroterghi liberi nudi, senza pubescenza. Edeago figg. 17-18, spermateca fig. 19.

\section{Comparazioni:}

La nuova specie è differente da O. tuberculatus Bernhauer, 1908, pure del Brasile, perché il quinto urotergo libero del maschio non presenta quattro lunghe carene come in tuberculatus.

Etimologia: La nuova specie per la sua taglia corporea ridotta e per l'assenza di caratteri differenziali vistosi è chiamato «modesto o senza pretese». 


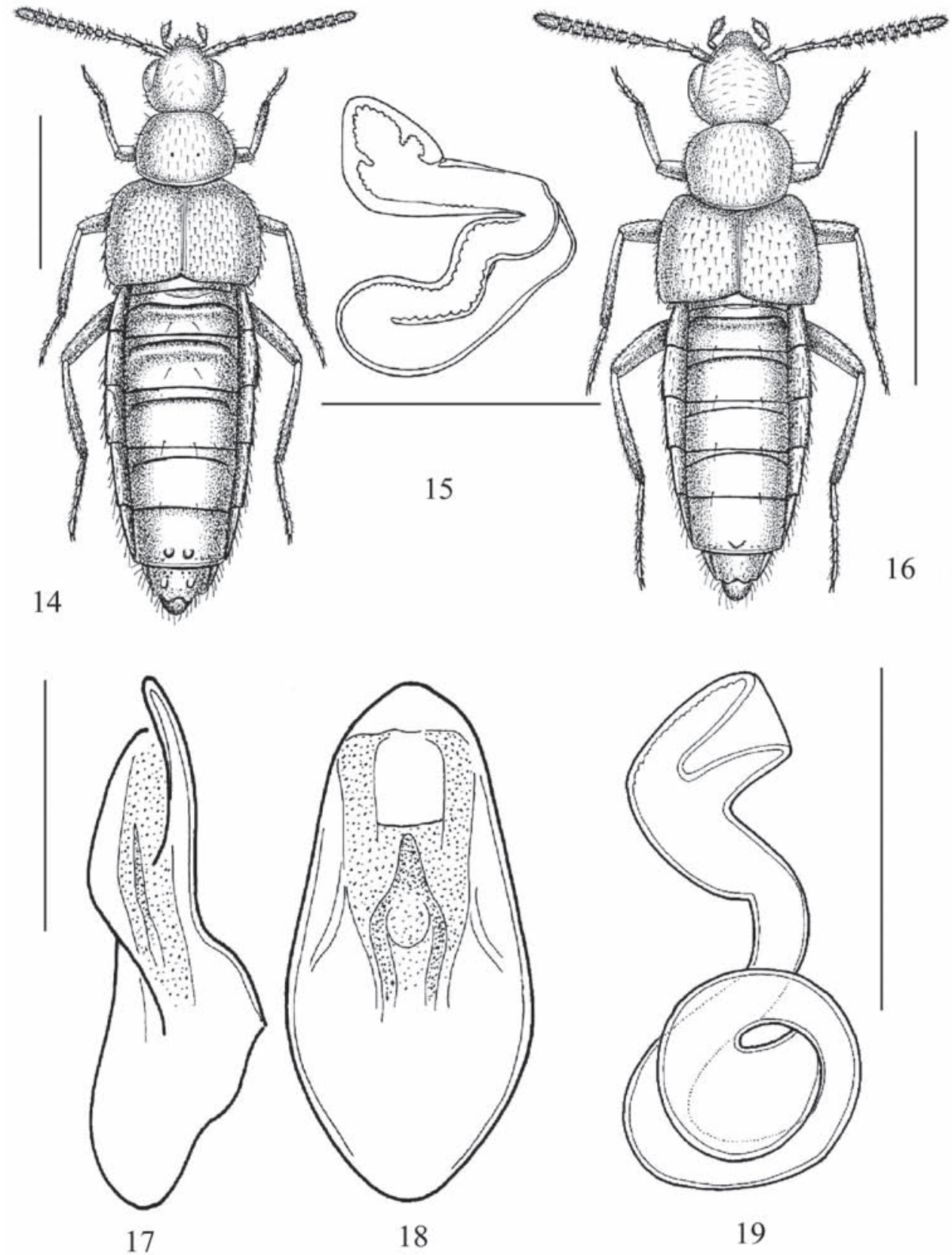

Figg. 14-19: Habitus, spermateca e edeago in visione laterale e ventrale. 14-15. Orphnebius caligatus n. sp.; 16-19. Orphnebius modestus $\mathrm{n}$. sp. Habitus scala $1 \mathrm{~mm}$, altre scale $0,1 \mathrm{~mm}$. 


\section{Orphnebius rioensis n. sp.}

(Figg. 20-22)

Materiale tipico: Holotypus ${ }^{\star}$, Rio Jan. Sta. Cruz, leg. Dr. Hensel (MB).

Descrizione:

Lunghezza 2,2 mm. Corpo lucidissimo e giallo-rossiccio con capo bruno; antenne e zampe giallo-rossicci. Punteggiatura del capo e del pronoto assente. Granulosità delle elitre saliente. Reticolazione del capo molto superficiale, quella del pronoto, elitre e addome assente. Uroterghi liberi nudi, senza pubescenza. Una fossetta a ciascun lato del pronoto. Edeago figg. 21-22.

\section{Comparazioni:}

La nuova specie è differente da O. tuberculatus BERNHAUER, 1908, pure del Brasile, per il differente colore del corpo, giallo-rossiccio con capo bruno nella nuova specie e nero pece con elitre nerobruno e capo e pronoto di un nero profondo in tuberculatus.

Etimologia: La nuova specie prende nome dalla grande città di Rio de Janeiro.

\section{Orphnebius transversus n. sp.}

(Figg. 23-24)

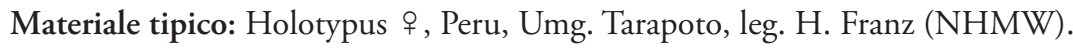

\section{Descrizione:}

Lunghezza 1,7 mm. Corpo lucido e bruno-rossiccio; antenne brune con i tre antennomeri basali gialli e quarto e quinto giallo-bruni; zampe giallo-rossicce. Granulosità dell'avancorpo fine. Corpo senza reticolazione. Uroterghi liberi nudi, senza pubescenza. Sul capo un punto discale evidente. Spermateca fig. 24.

\section{Comparazioni:}

La nuova specie è simile a $O$. pungitius Solsky, 1875, pure del Perù, ma il pronoto è più largo del capo (pronoto non più largo del capo in pungitius) e le elitre non hanno una carena laterale come nelle elitre di pungitius.

Etimologia: La nuova specie prende nome di «trasverso» a motivo del pronoto appunto molto trasverso.

\section{Orphnebius tarapotensis n. sp.}

(Figg. 25-27)

Materiale tipico: Holotypus $0^{\star}$, Peru, Umg. Tarapoto, leg. H. Franz (NHMW).

\section{Descrizione:}

Lunghezza 1,8 mm. Corpo lucido e bruno-rossiccio con primo urotergo libero giallo-bruno; antenne brune con antennomero basale rossiccio e undicesimo giallo; zampe gialle con femori posteriori bruni fino oltre la metà dello stesso femore. Punteggiatura del capo e del pronoto assente. Granulosità delle elitre superficiale. Corpo senza reticolazione. Uroterghi liberi nudi, senza pubescenza. Sesto urotergo libero del maschio con quattro lobi al margine posteriore, i laterali più stretti degli interni. Edeago figg. 26-27. 

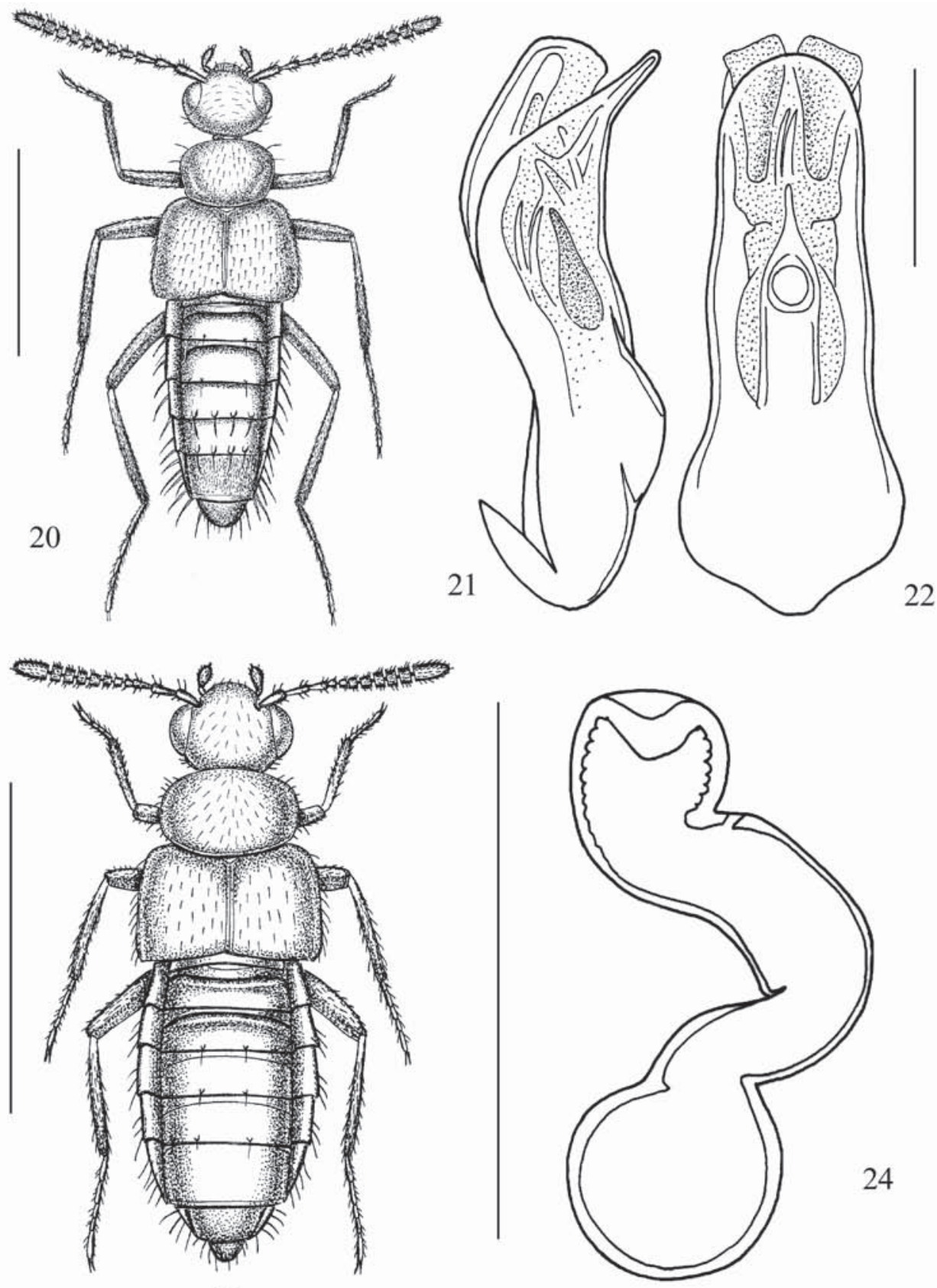

23

Figg. 20-24: Habitus, edeago in visione laterale e ventrale e spermateca. 20-22. Orphnebius rioensis n. sp.; 23-24. Orphnebius transversus $\mathrm{n}$. sp. Habitus scala $1 \mathrm{~mm}$, altre scale $0,1 \mathrm{~mm}$. 


\section{Comparazioni:}

La nuova specie è nettamente differente da O. pungitius Solsky, 1875, pure del Perù, per il differente colore del corpo, per il pronoto poco trasverso (pronoto molto trasverso in pungitius) e per l'assenza di impressione sulla metà anteriore del pronoto, presenti al contrario su quello di pungitius.

Etimologia: La nuova specie prende nome dalla città peruviana di Tarapoto.

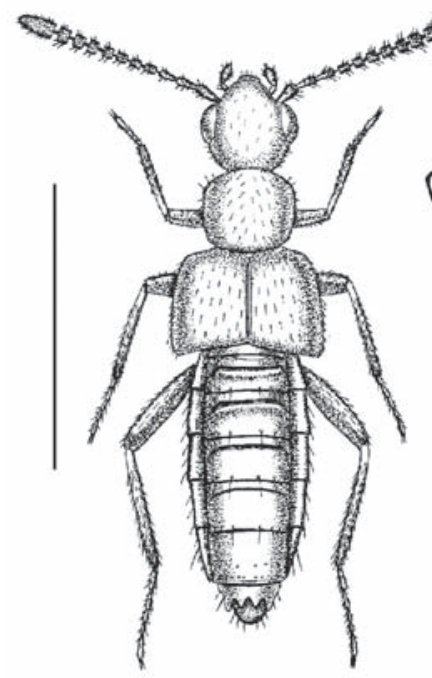

25

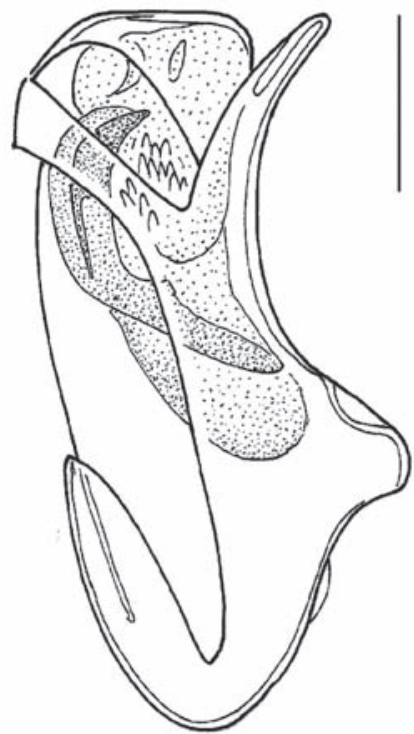

29

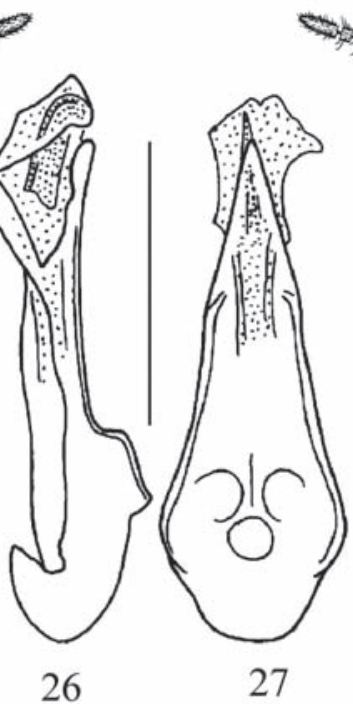

27
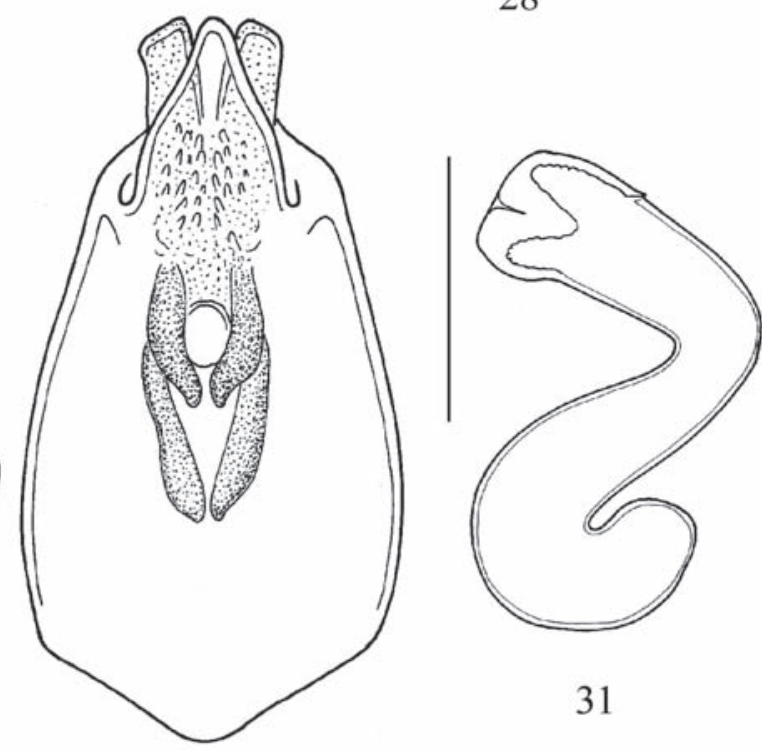

30

\section{8}

Figg. 25-31: Habitus, edeago in visione laterale e ventrale e spermateca. 25-27. Orphnebius tarapotensis n. sp.; 28-31. Apalonia pernambucoensis $\mathrm{n}$. sp. Habitus scala $1 \mathrm{~mm}$, altre scale $0,1 \mathrm{~mm}$. 


\section{Apalonia pernambucoensis n. sp.}

(Figg. 28-31)

Materiale tipico: Holotypus $\sigma^{\star}$, Bras., Pernambuco, 17.XI.1886, leg. Fruhstorfer (MB).

Paratypi: $10^{\star}$ e 1 옹 stessa provenienza (MB), (MRSNT).

\section{Descrizione:}

Lunghezza 2,5 mm. Corpo lucido e bruno-rossiccio con uroterghi liberi secondo e terzo bruni; antenne giallo-rossicce, verso l'estremità distale rossicce; zampe giallo-rossicce. Punteggiatura del capo, del pronoto e delle elitre fine e superficiale. Reticolazione del capo presente, resto del corpo senza reticolazione. Uroterghi liberi nudi, senza pubescenza. Sesto urotergo libero del maschio solcato longitudinalmente da strie sulla metà posteriore. Edeago figg. 29-30, spermateca fig. 31 .

\section{Comparazioni:}

Per la forma della spermateca e dell'edeago, la nuova specie si pone tassonomicamente vicino ad A. glabricollis (Bernhauer, 1940), della Costa Rica (serie tipica del DEI da me esaminata). Ma nella nuova specie la reticolazione del capo è superficiale, mentre è forte in glabricollis, l'edeago è tozzo nella nuova specie e snello in glabricollis, e la porzione prossimale della spermateca descrive uno stretto semicerchio, mentre è ampio in glabricollis.

Etimologia: La nuova specie prende nome dalla città di Pernambuco in Brasile.

\section{Apalonia tupiorum n. sp.}

(Figg. 32-35)

Materiale tipico: Holotypus ${ }^{\star}$, Brasilien, Sta. Catharina, Theresopolis, leg. S. Fruhstorfer (MB).

Paratypi: 1 ㅊ e 2 우 오, Brasilien, Sta. Catharina, Lages Hochlands, leg. S. Fruhstorfer (MB), (MRSNT).

\section{Descrizione:}

Lunghezza 2,5 mm. Corpo lucido e bruno-rossiccio; antenne bruno-rossicce con i tre antennomeri basali giallo-rossicci; zampe giallo-rossicce. Granulosità del capo e del pronoto fine e saliente, quella delle elitre assente. Reticolazione del capo forte, quella del pronoto e delle elitre superficiale, quella dell'addome manca. Uroterghi liberi nudi, senza pubescenza. Sesto urotergo libero del maschio attraversato trasversalmente da uno spigolo sinuato saliente. Edeago figg. 33-34, spermateca fig. 35 .

\section{Comparazioni:}

L'habitus della nuova specie è simile a quello di A. glabricollis (Bernhauer, 1940), della Costa Rica (serie tipica del DEI da me esaminata), ma l'apice distale dell'edeago della nuova specie, in visione ventrale, è assai stretto, largo metà che in glabricollis. La spermateca è simile nelle due specie, ma è snella nella nuova specie e tozza in glabricollis.

Etimologia: La nuova specie prende nome dal locale gruppo etnico brasiliano dei Tupì. 


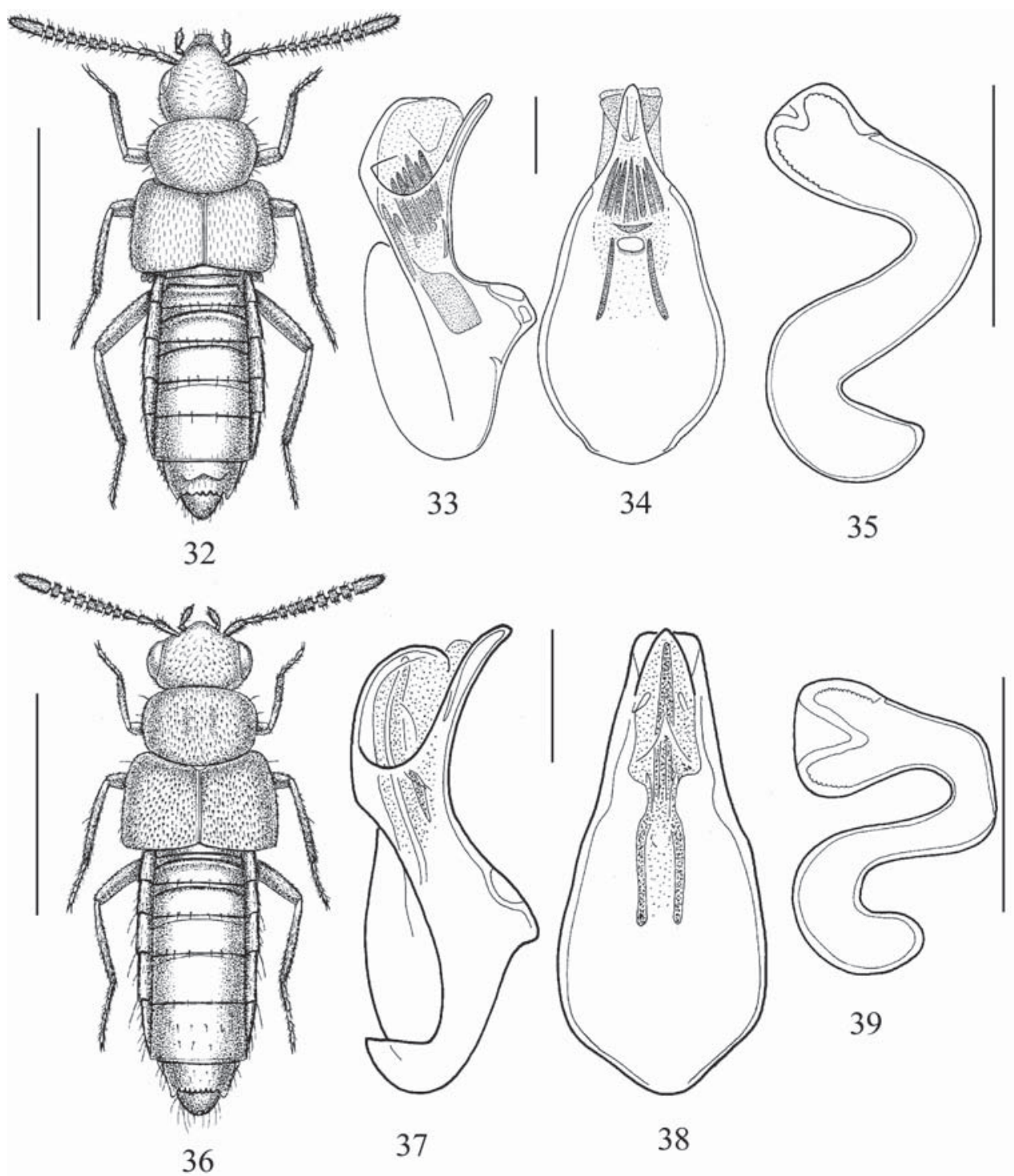

Figg. 32-39: Habitus, edeago in visione laterale e ventrale e spermateca. 32-35. Apalonia tupiorum n. sp.; 36-39. Apalonia obscura n. sp. Habitus scala $1 \mathrm{~mm}$, altre scale $0,1 \mathrm{~mm}$.

\section{Apalonia obscura n. sp.}

(Figg. 36-39)

Materiale tipico: Holotypus ơ , Brasil, Espirito Santo, Sta. Thereze, XI.1929, leg. O. Conde (MB).

Paratypi: 32 es., stessa provenienza (MB), (MRSNT). 


\section{Descrizione:}

Lunghezza $2 \mathrm{~mm}$. Corpo lucido e nero-bruno; antenne brune con i due antennomeri basali giallorossicci; zampe giallo-rossicce. Punteggiatura del capo e delle elitre evidente, quella del pronoto superficiale. Reticolazione del capo e delle elitre molto superficiale, quella del pronoto evanescente. Uroterghi liberi nudi, senza pubescenza, tranne che sul quinto urotergo libero su cui è rada. Margine posteriore del sesto urotergo libero del maschio crenellato. Edeago figg. 37-38, spermateca fig. 39.

\section{Comparazioni:}

Gli occhi molto sviluppati e il quarto antennomero molto trasverso sono caratteri che distinguono esternamente la nuova specie da A. glabricollis (Bernhauer, 1940), della Costa Rica (serie tipica del DEI da me esaminata), che presenta occhi meno sviluppati e quarto antennomero lungo quanto largo. L'edeago è minore nella nuova specie e maggiore in glabricollis e l'introflessione apicale del bulbo distale della spermateca è larga e prolungata nella nuova specie e breve e stretta in glabricollis.

Etimologia: La nuova specie prende nome di «oscura» per il colore nero-bruno del suo corpo.

\section{Apalonia debilis n. sp.}

(Figg. 40-41)

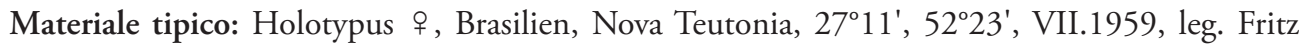
Plaumann (MB).

\section{Descrizione:}

Lunghezza 2,3 mm. Corpo lucido e giallo-bruno con addome giallo-bruno ad uroterghi liberi quarto e quinto bruni; antenne rossicce con i tre antennomeri basali giallo-rossicci; zampe giallorossicce. Punteggiatura del capo evidente, ma assente sulla fascia longitudinale mediana, quella del pronoto quasi invisibile e quella delle elitre molto superficiale e fine. Sono presenti granuli salienti solo al margine posteriore degli uroterghi liberi. Corpo senza reticolazione. Uroterghi liberi nudi, senza pubescenza. Spermateca fig. 41.

\section{Comparazioni:}

La spermateca della nuova specie è simile a quella di A. semiscapa (PACE, 1987), comb. n., di Guadalupa («olim» Macrogerodonia semiscapa PACE, 1987: 194), ma il bulbo distale della spermateca è reniforme nella nuova specie e ovale in semiscapa. Il pronoto, inoltre, è molto trasverso nella nuova specie e lievemente trasverso in semiscapa.

Etimologia: Il nome della nuova specie significa «debole».

\section{Apalonia laboriosa n. sp.}

(Figg. 42-44)

Materiale tipico: Holotypus ${ }^{\star}$, Paraguagy, San Bernardino, 1905, leg. K. Fiebrig (MB).

\section{Descrizione:}

Lunghezza 2,8 mm. Corpo lucido e giallo con capo bruno-rossiccio, elitre giallo-rossicce e uroterghi liberi terzo, quarto e base del quinto bruni; antenne e zampe gialle. Granulosità del capo 
superficiale, ma saliente sulla fronte, quella del pronoto ben visibile e quella delle elitre molto superficiale. Reticolazione del capo e delle elitre molto evanescente, reticolazione del pronoto forte. Uroterghi liberi nudi, senza pubescenza. Capo largamente impresso sul disco. Sesto urotergo libero del maschio con margine posteriore crenellato. Edeago figg. 43-44.

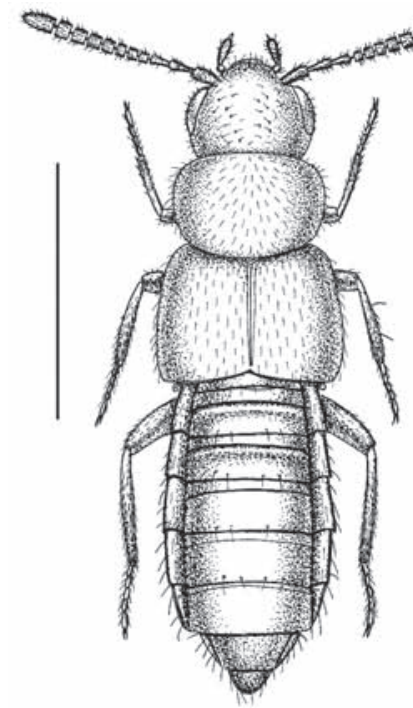

40
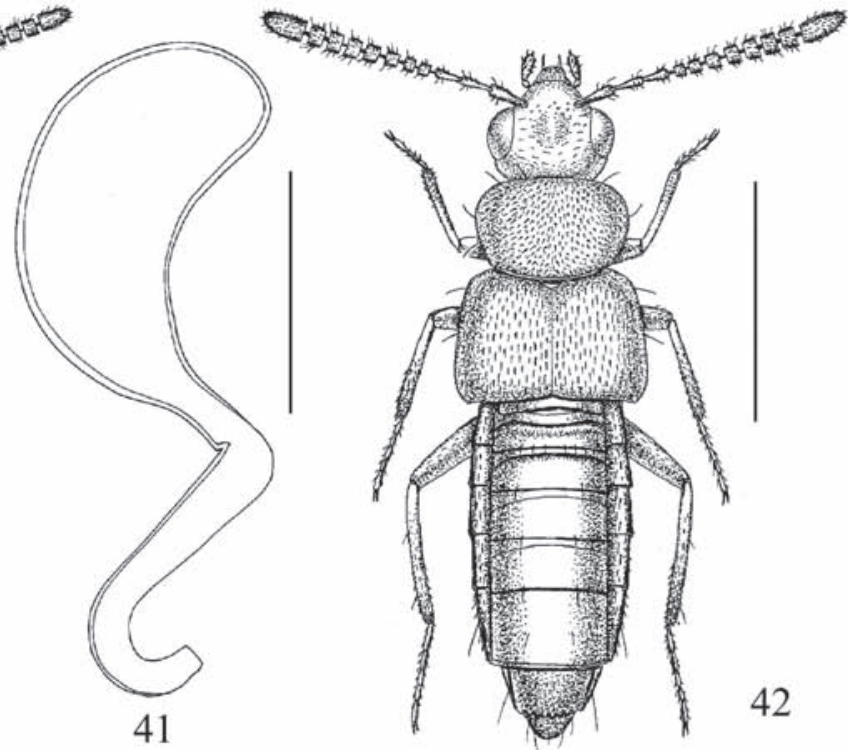

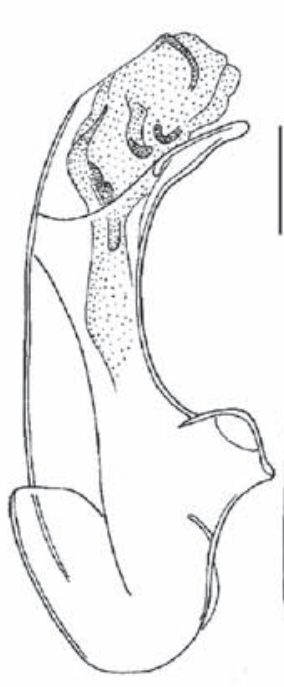

43

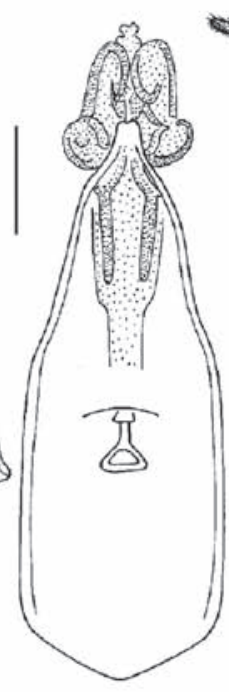

44

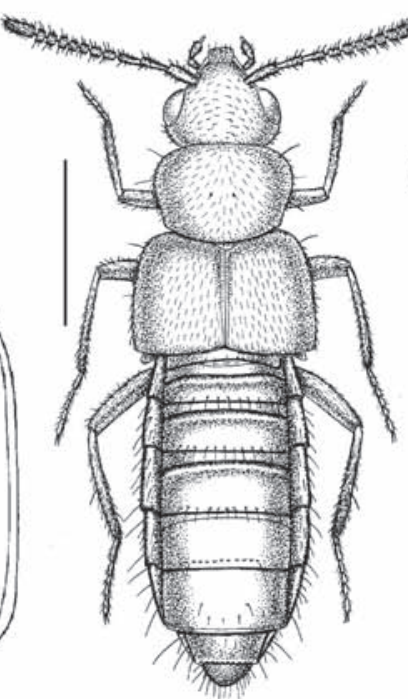

45

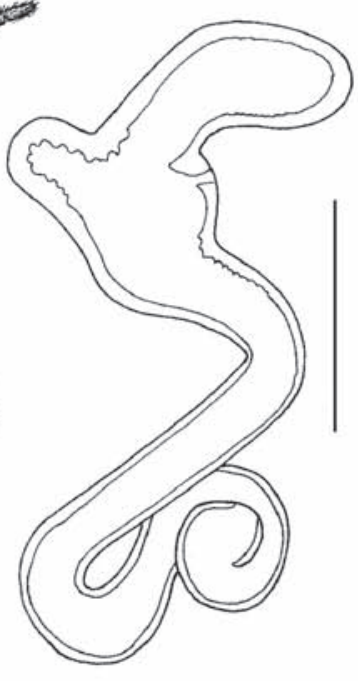

46

Figg. 40-46: Habitus, spermateca e edeago in visione laterale e ventrale. 40-41. Apalonia debilis n. sp.; 42-44. Apalonia laboriosa n. sp.; 45-46. Apalonia seminigra n. sp. Habitus scala $1 \mathrm{~mm}$, altre scale $0,1 \mathrm{~mm}$. 


\section{Comparazioni:}

L'edeago della nuova specie ha forma simile a quella dell'edeago di A. circumflexa PACE, 1977, dell'Ecuador, ma l'armatura genitale interna della nuova specie è esile, robusta in circumflexa, e la porzione apicale distale dell'edeago, in visione ventrale, ha lati sinuati nella nuova specie e rettilinei in circumflexa.

Etimologia: La nuova specie prende nome di «faticosa» perché ha richiesto faticoso lavoro per la sua determinazione.

\section{Apalonia seminigra n. sp.}

(Figg. 45-46)

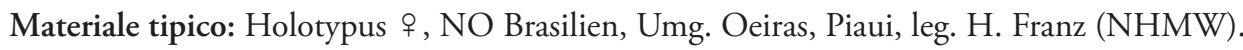

\section{Descrizione:}

Lunghezza 3,7 mm. Corpo lucido con capo e pronoto neri, elitre giallo-brune e addome rossiccio con gli uroterghi liberi terzo, quarto e quinto bruno-rossicci; antenne e zampe gialle. Punteggiatura del capo e del pronoto forte, quella delle elitre fine ed evidente. Corpo senza reticolazione. Uroterghi liberi nudi, senza pubescenza. Sesto urotergo libero del maschio con margine posteriore crenellato. Spermateca fig. 46.

\section{Comparazioni:}

La spermateca della nuova specie ha forma che ricorda da lontano quella di $A$. fuscofemoralis PACE, 1997, della Colombia. Se ne distingue per la presenza di una sinuosità del bulbo distale, assente in fuscofemoralis. Gli antennomeri sesto a decimo sono fortemente trasversi, mentre in fuscofemoralis sono appena trasversi.

Etimologia: La nuova specie ha solo il capo e il pronoto neri, per questo è chiamata «mezza nera».

\section{Apalonia maculifrons $\mathbf{n}$. sp.}

(Figg. 47-50)

Materiale tipico: Holotypus $\sigma^{\star}$, NO Brasilien, Umg. Oeiras, Piaui, leg. H. Franz (NHMW).

Paratypi: 2 es., stessa provenienza (MRSNT).

\section{Descrizione:}

Lunghezza $3 \mathrm{~mm}$. Corpo lucido con capo nero con grande macchia giallo-rossiccia tra l'inserzione delle antenne, pronoto giallo-rossiccio, elitre giallo-brune con omeri gialli, uroterghi liberi basali primo e secondo giallo-rossicci, restanti uroterghi liberi neri; antenne giallo-brune con i tre antennomeri basali e metà apicale dell'undicesimo rossicci; zampe giallo-rossicce. Punteggiatura del capo e pronoto molto superficiale. Granulosità delle elitre molto evanescente. Corpo senza reticolazione, tranne che sul pronoto coperto di reticolazione molto superficiale. Uroterghi liberi nudi, senza pubescenza. Edeago figg. 48-49, spermateca fig. 50. 

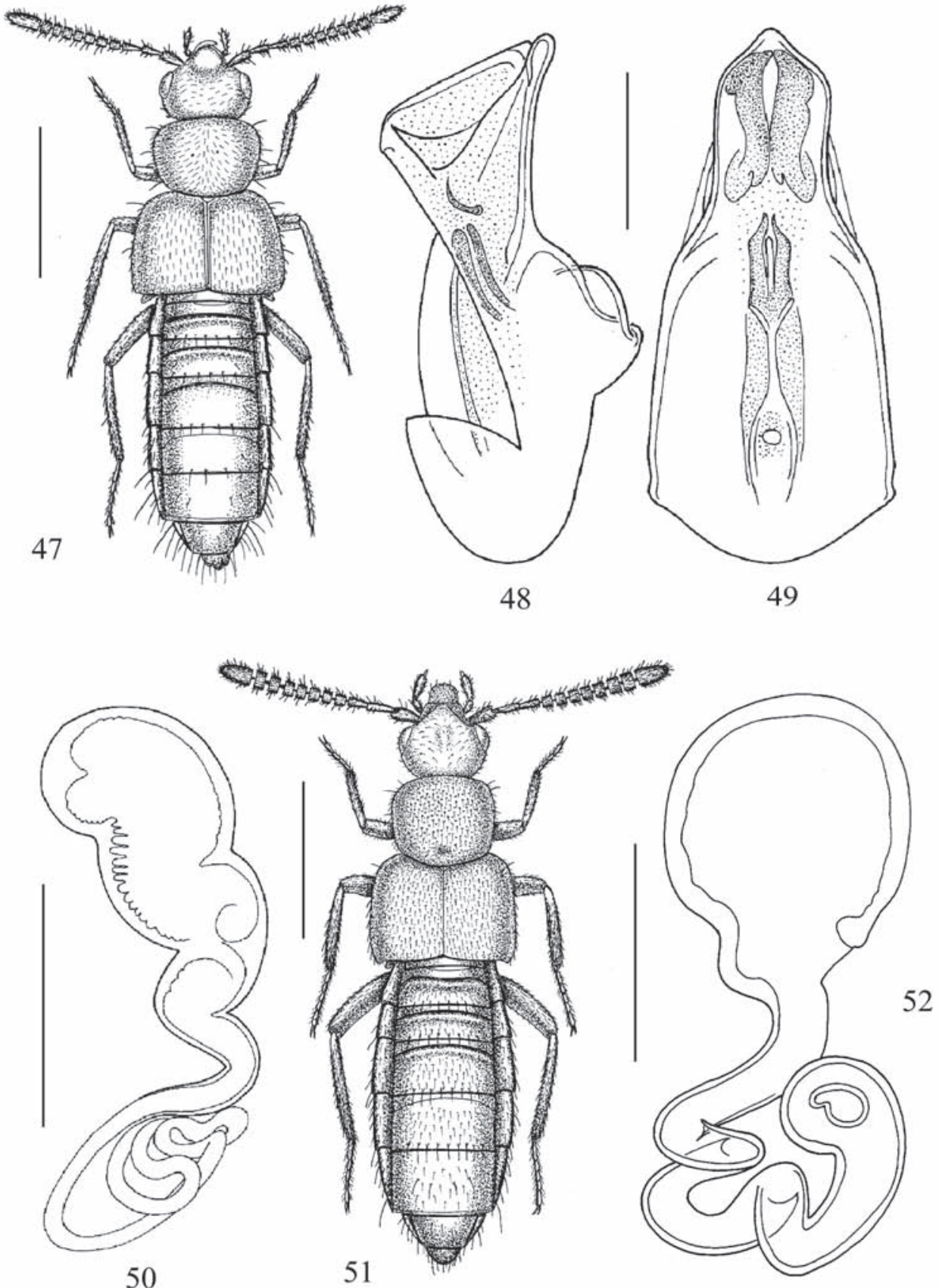

Figg. 47-52: Habitus, edeago in visione laterale e ventrale e spermateca. 47-50. Apalonia maculifrons n. sp.; 51-52. Apalonia catharinensis $\mathrm{n}$. sp. Habitus scala $1 \mathrm{~mm}$, altre scale $0,1 \mathrm{~mm}$. Habitus scala $1 \mathrm{~mm}$, altre scale $0,1 \mathrm{~mm}$. 


\section{Comparazioni:}

La nuova specie presenta la porzione prossimale della spermateca avvolta a spire come in A. bondensis PaCe, 1997, della Colombia, ma in essa le spire sono ampie e di numero ridotto, mentre in bondensis sono strette e numerose. Il pronoto, inoltre, è meno trasverso di quello di bondensis e giallo-rossiccio, mentre in bondensis è giallo-bruno.

Etimologia: La nuova specie è chiamata «macchia sulla fronte» per la presenza di questo suo carattere differenziale.

\section{Apalonia catharinensis n. sp.}

(Figg. 51-52)

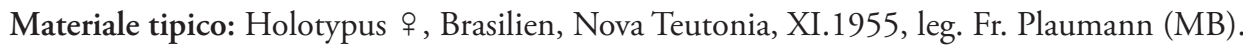

\section{Descrizione:}

Lunghezza 3,8 mm. Avancorpo lievemente opaco, addome lucido. Corpo bruno-rossiccio con quarto urotergo libero bruno; antenne brune con i tre antennomeri basali giallo-rossicci; zampe giallo-rossicce. Granulosità del capo e del pronoto fine e saliente, quella delle elitre molto superficiale e fine. Reticolazione del capo e delle elitre superficiale, quella del pronoto evidente e quella dell'addome molto trasversa, fine e superficiale. Capo con solco mediano frontale Spermateca fig. 52 .

\section{Comparazioni:}

La nuova specie presenta pronoto poco trasverso e porzione prossimale della spermateca avvolta in spire come in A. fuscofemoralis PACE, 1997, della Colombia. La nuova specie si distingue da questa per il bulbo distale della spermateca subsferico, mentre è botuliforme (a forma di salsiccia) in fuscofemoralis e il quarto antennomero è trasverso nella nuova specie e più lungo che largo in fuscofemoralis.

Etimologia: La nuova specie prende nome dalla regione di Santa Catharina in cui si trova la località tipica Nova Teutonia.

\section{Apalonia marginella PACE, 1997}

Apalonia marginella PACE, 1997: 25

1 우, Brasilien, Sta. Catharina, vor 1930, leg. W. Ehrhardt (MB), Figg. 53-54.

Distribuzione: Specie già nota della Colombia. Nuova per il Brasile.

\section{Myrmecoxenia regia n. sp.}

(Figg. 55-56)

Materiale tipico: Holotypus $ᄋ$, Brasilien, Nova Teutonia, VI.1960, leg. F. Plaumann (MB).

\section{Descrizione:}

Lunghezza 4,5 mm. Corpo lucidissimo e bruno-rossiccio con addome rossiccio; antenne brunorossicce con antennomeri basali secondo a quinto rossicci; zampe rossicce. Granulosità del capo e 
pronoto fine e saliente. Granuli delle elitre forti e salienti. Corpo interamente senza reticolazione. Pronoto con debole solco mediano e con due forti punti mediani disposti trasversalmente. Lato esterno delle elitre carenato. Quinto urotergo libero della femmina con debole tubercolo mediano posteriore tra una striatura longitudinale che all'indietro diviene sempre più fitta, così da dare una aspetto opaco alla superficie. Margine posteriore del sesto urotergo libero della femmina con quattro larghi denti triangolari. Spermateca fig. 56.

\section{Comparazioni:}

La nuova specie è distinta da $M$. pampeana Lynch-Arribalzaga, 1884, dell'Argentina, per il capo più stretto del pronoto (capo largo quanto il pronoto in pampeana), per il colore brunorossiccio del capo (capo giallo-rossiccio, ma nero pece sul disco e sulla fronte in pampeana), per il terzo antennomero poco più lungo del secondo (quasi metà più lungo del secondo in pampeana), per il pronoto trasverso e senza solco basale trasverso (lungo quanto largo in pampeana e con solco trasverso piuttosto forte alla base), per le elitre nettamente più lunghe del pronoto e non poco più lunghe del pronoto come in pampeana.

Etimologia: La nuova specie prende nome di «regale» per l'eleganza del suo habitus.

\section{Macrogerodonia foveaventer n. sp.}

(Figg. 57-59)

Materiale tipico: Holotypus ơ , Colombia, Muzo (MB).

\section{Descrizione:}

Lunghezza $10 \mathrm{~mm}$. Corpo lucido e giallo-rossiccio (esemplare immaturo), occhi neri, quarto urotergo libero del maschio bruno-rossiccio; antenne bruno-rossicce con i quattro antennomeri basali giallo-rossicci; zampe gialle. Punteggiatura del capo appena evanescente e assente sulla fascia longitudinale mediana, quella del pronoto evidente e quella delle elitre superficiale. orpo privo di reticolazione. Pronoto con profonda impressione a ciascun lato, una larga depressione mediana discale e una profonda fossetta mediana posteriore trasversa. Superficie delle elitre ondulata. Un solo solco trasverso basale sul primo urotergo libero del maschio. Margine posteriore dello stesso lobato posteriormente con una spina mediana marginale. Terzo urotergo libero del maschio con ampia e poco profonda concavità o fovea basale. Quarto urotergo libero del maschio con profondissima fovea mediana basale. Quinto urotergo libero del maschio con un gradino longitudinale a ciascun lato. Sesto urotergo libero del maschio con forti granuli. Edeago figg. 58-59.

\section{Comparazioni:}

La nuova specie è simile a $M$. peruviana (Bernhauer, 1908), del Perù, (holotypus o t da me esaminato. Bernhauer afferma che è un $\sigma^{\star}$, ma non ha dissezionato l'esemplare), soprattutto per il grande sviluppo oculare, la forma del pronoto con tutte le sue impressioni e per il margine laterale del sesto urotergo libero seghettato. La nuova specie se ne distingue per le elitre giallo-rossicce e superficialmente punteggiate, mentre in peruviana le elitre sono gialle con macchia laterale bruna e coperte di forti granuli, il margine posteriore del terzo urotergo libero è giallo-rossiccio nella nuova specie e bruno in peruviana.

Etimologia: La nuova specie prende nome di «buca sull'addome» appunto per la presenza di una profonda incavatura mediana del quarto urotergo libero del maschio. 

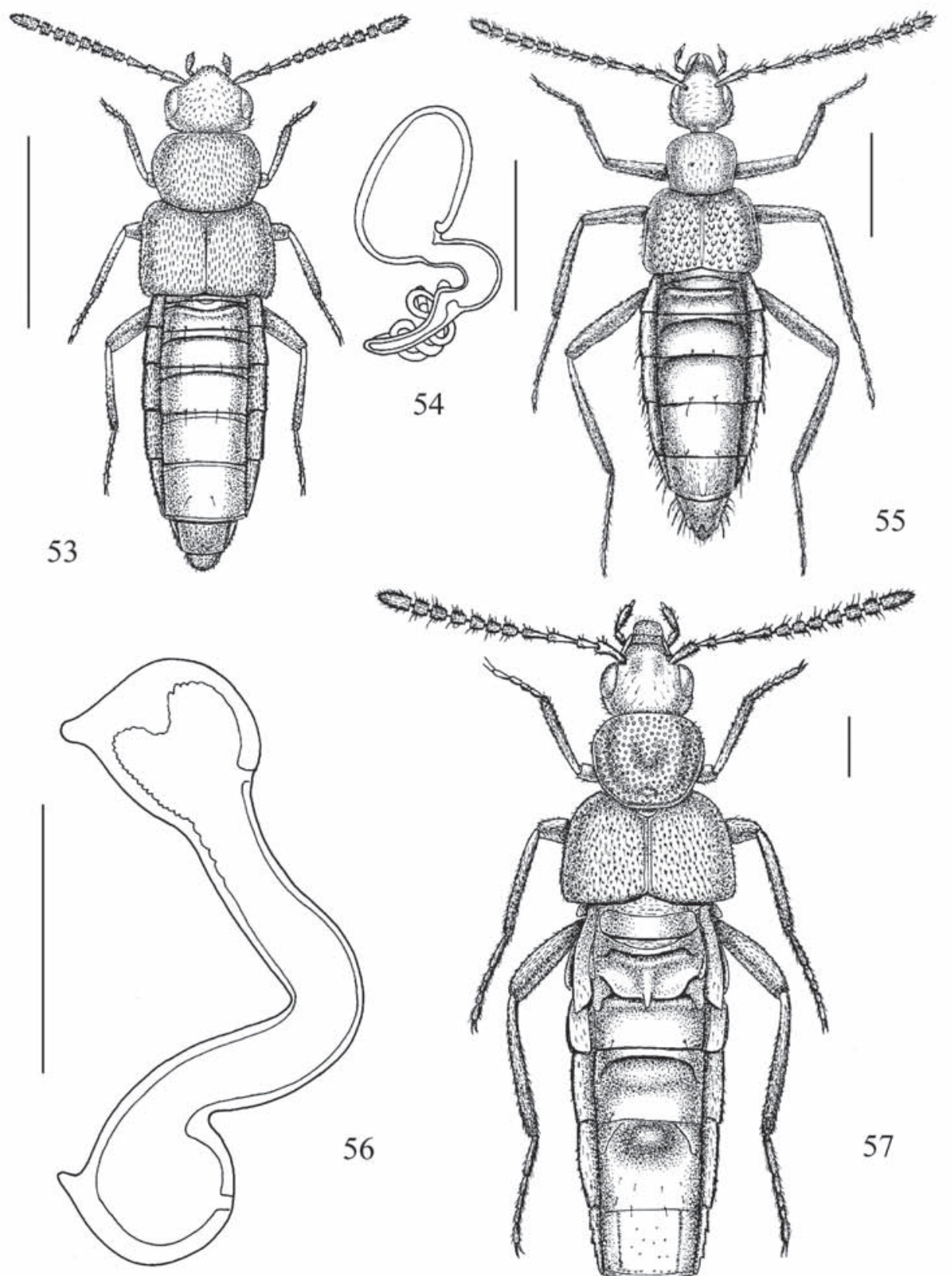

56

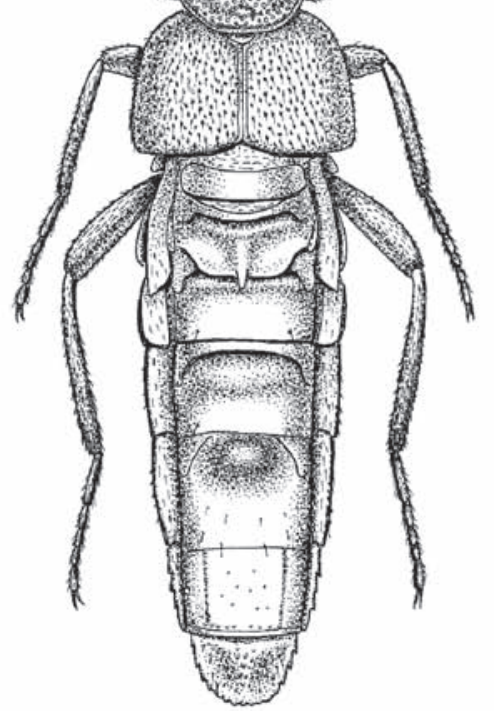

Figg. 53-57: Habitus e spermateca. 53-54. Apalonia marginella PACE, 1997; 55-56. Myrmecoxenia regia n. sp.; 57. Macrogerodonia foveaventer n. sp. Habitus scala $1 \mathrm{~mm}$, altre scale $0,1 \mathrm{~mm}$. Habitus scala $1 \mathrm{~mm}$, altre scale $0,1 \mathrm{~mm}$. 


\section{Macrogerodonia paraensis n. sp.}

(Figg. 60-62)

Materiale tipico: Holotypus ơ , Brasilien, Para, Umg. Belem, Umg. Capitao Poco, Juni 1983, leg. H. Franz (NHMW).

\section{Descrizione:}

Lunghezza 5,4 mm. Corpo lucido con avancorpo bruno-rossiccio e addome giallo-rossiccio; antenne bruno-rossicce con i tre antennomeri basali e l'undicesimo giallo-rossicci; zampe giallorossicce. Punteggiatura del capo superficiale. Granuli del pronoto forti sono diffusi fino allo spigolo trasverso prebasale, oltre il quale non si trovano granuli. Granulosità delle elitre grossolana e assai saliente, assente sulla fascia perimarginale trasversa posteriore. Uroterghi liberi nudi con quinto urotergo libero del maschio punteggiato sulla metà anteriore e striato longitudinalmente su quella posteriore con granuli allungati interposti. Punteggiatura e striatura non sono presenti ai lati di questo urotergo libero del maschio. Sesto urotergo libero del maschio opaco tranne ai lati, con forti granuli. Edeago figg. 61-62.

\section{Comparazioni:}

La nuova specie è chiaramente distinta da M. magnicollis Bernhauer, 1941, del Perù (holotypus $o^{\star}$ da me esaminato. Il pronoto di questo esemplare è perduto). Se ne distingue per gli antennomeri quarto a decimo più lunghi che larghi (trasversi in magnicollis) e per le elitre fortemente granulose su tutta la superficie, tranne al margine posteriore, mentre in magnicollis la forte granulosità è presente solo sulla fascia perisuturale, assente sul resto delle elitre. L'edeago della nuova specie è arcuato all'apice distale, in visione ventrale, e appuntito in magnicollis.

Etimologia: La nuova specie prende nome dalla regione del Para.

\section{Macrogerodonia boliviana n. sp.}

(Figg. 63-64)

Materiale tipico: Holotypus ^ , Bolivien, Umg. Sta. Cruz, leg. H. Franz (NHMW).

\section{Descrizione:}

Lunghezza $4 \mathrm{~mm}$. Corpo lucido e giallo-rossiccio con capo bruno-rossiccio; antenne e zampe giallo-rossicce. Punteggiatura del capo forte e fine, assente sulla fascia longitudinale mediana. Granulosità del pronoto superficiale. Granuli delle elitre forti solo sui due terzi anteriori, all'indietro gradualmente deboli fino ad assenti. Carena laterale su ciascuna elitra interrotta posteriormente. Uroterghi liberi della femmina nudi, quinto urotergo libero della femmina con punteggiatura evidente e granuli forti allineati obliquamente. Reticolazione assente su tutto il corpo. Spermateca fig. 64.

\section{Comparazioni:}

La nuova specie presenta habitus simile a quello di $M$. magnicollis Bernhauer, 1941, del Perù (holotypus $\sigma^{\star}$ da me esaminato). Se ne distingue per gli antennomeri quarto a decimo più lunghi che larghi (trasversi in magnicollis) e per gli uroterghi liberi primo a quarto quasi nudi, mentre in magnicollis sul secondo e terzo urotergo libero si trova una fila trasversa di punti.

Etimologia: La nuova specie prende nome dalla Bolivia. 


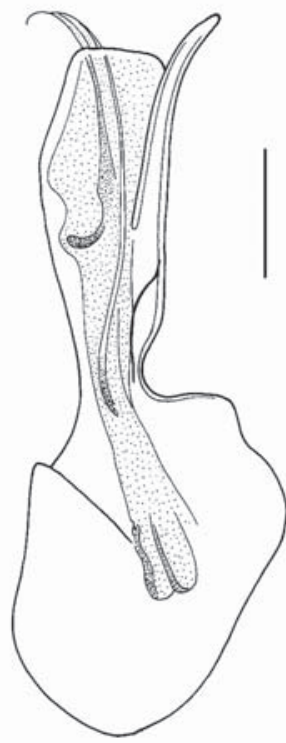

58

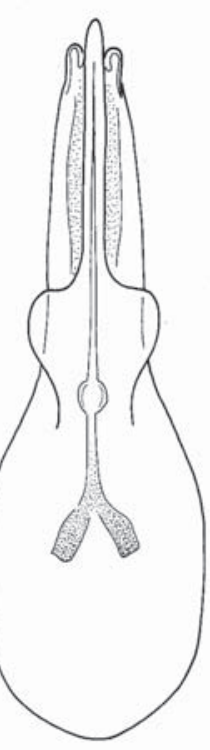

59
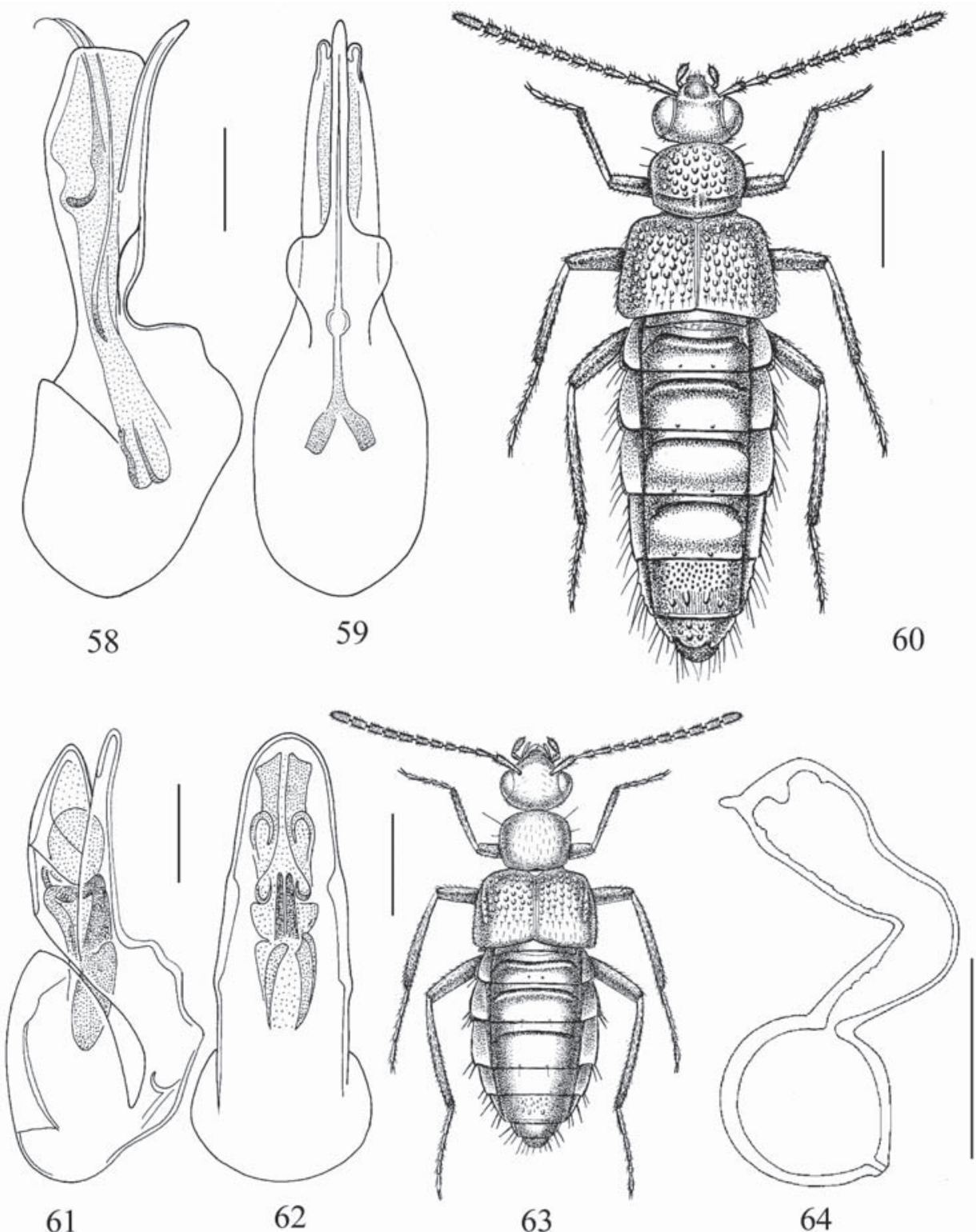

Figg. 58-64: Edeago in visione laterale e ventrale, habitus e spermateca. 58-59. Macrogerodonia foveaventer n. sp.; 60-62. Macrogerodonia paraensis n. sp.; 63-64. Macrogerodonia boliviana $\mathrm{n}$. sp. Habitus scala $1 \mathrm{~mm}$, altre scale $0,1 \mathrm{~mm}$. Habitus scala $1 \mathrm{~mm}$, altre scale $0,1 \mathrm{~mm}$.

\section{Macrogerodonia unidens $\mathbf{n}$. sp.}

(Figg. 65-67)

Materiale tipico: Holotypus ${ }^{\star}$, Brasilien, Rio Grande do Sul, Umg. Sta. Maria, leg. H. Franz (NHMW). 


\section{Descrizione:}

Lunghezza 5,7 mm. Avancorpo opaco, addome debolmente lucido. Corpo nero con macchia rossiccia al margine posteriore dei paratergiti e pigidio bruno-rossiccio; antenne bruno-rossicce con i tre antennomeri basali e apice dell'undicesimo giallo-rossicci. Punteggiatura del capo forte, quella delle elitre d'aspetto rugoso. Granulosità del pronoto fittissima e saliente, assente sulla fascia longitudinale mediana. Primo urotergo libero del maschio protratto all'indietro a forma di triangolo, tale espansione coperta di granulosità saliente. Granulosità del secondo urotergo libero saliente, quella dei restanti uroterghi liberi superficiale. Reticolazione del capo e del pronoto forte e finissima, quella delle elitre evidente e quella dell'addome finissima e ben visibile. Fronte con due carene salienti convergenti verso il labbro. Pronoto con debole impressione a ciascun lato e con ampia concavità mediana. Quinto urotergo libero del maschio con tubercolo mediano posteriore gradualmente saliente all'indietro. Edeago figg. 66-67.

\section{Comparazioni:}

La nuova specie è simile a $M$. longicollis PACE, 1986, del Perù, per avere un dente mediano al margine posteriore del primo urotergo libero del maschio. Se ne distingue per la granulosità fitta del pronoto, mentre in longicollis il pronoto è privo di punteggiatura o granulosità. Gli antennomeri intermedi della nuova specie sono trasversi, mentre in longicollis sono più lunghi che larghi. La porzione apicale distale dell'edeago, in visione ventrale, è larga nella nuova specie e in longicollis strettissima.

Etimologia: Il nome della nuova specie significa «dente unico» a motivo della presenza di un solo dente mediano saliente sul quinto urotergo libero del maschio.

\section{Zyras (Diaulaconia) carinilabiatus n. sp.}

(Figg. 68-69)

Materiale tipico: Holotypus ㅇ, Bolivien, Prov. Sara, leg. J. Steinbach (MB).

\section{Descrizione:}

Lunghezza $9 \mathrm{~mm}$. Corpo debolmente opaco e giallo-rossiccio, con capo bruno-rossiccio; antenne e zampe rossicce. Punteggiatura del capo fine e superficiale, assente sul disco, quella del pronoto ed elitre assente. Granulosità dell'addome visibile. Reticolazione dell'avancorpo forte, quella del primo urotergo libero della femmina ben visibile, quella sui restanti uroterghi liberi finissima. Labbro superiore fortemente carenato sulla linea longitudinale mediana. E' presente un breve solco mediano tra l'inserzione delle antenne. Antennomeri terzo a undicesimo compressi lateralmente. Debole depressione a ciascun lato del pronoto. Spermateca fig. 69.

\section{Comparazioni:}

L'habitus della nuova specie è molto simile a quello delle specie del sottogenere Diaulaconia Bernhauer, 1928, diffuso nella regione orientale ed etiopica, come Zyras compressicornis Fauvel, 1905, di Giava, tuttavia la spermateca della nuova specie è del tutto differente da quella delle specie note: avvolta a matassa nella nuova specie e a capsula allungata nelle altre specie. Antennomeri quarto a decimo più lunghi che larghi nella nuova specie e trasversi o lunghi quanto larghi nelle specie a me note. Sia le antenne della nuova specie che quelle delle altre specie sono compresse lateralmente.

Etimologia: La nuova specie prende nome di «con labbro carenato» per la presenza di una vistosa carena mediana del labbro superiore. 


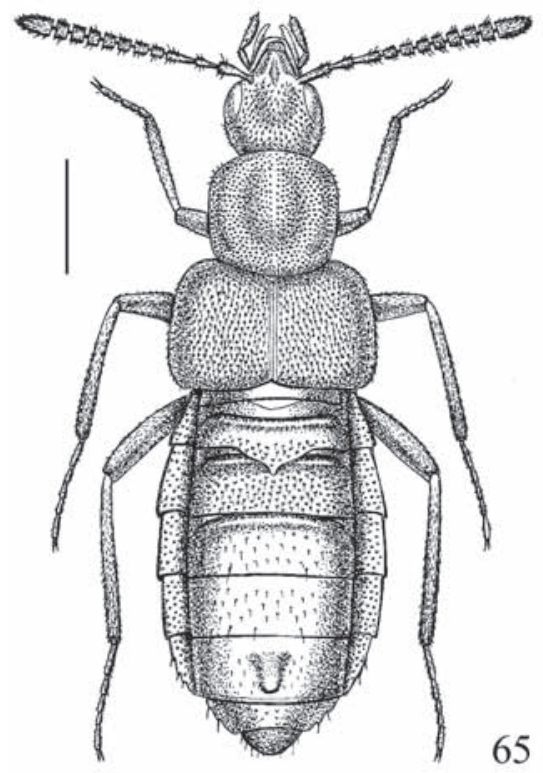

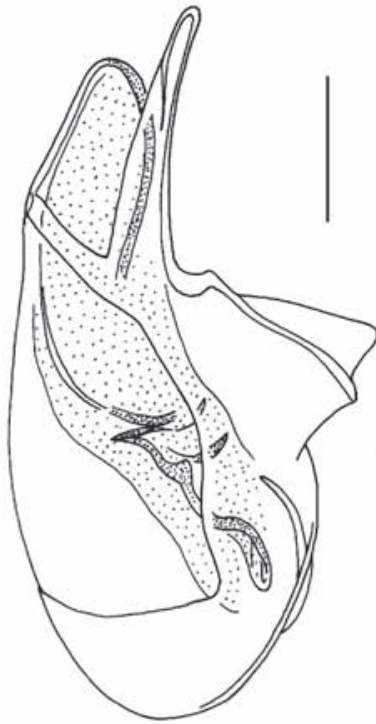

66

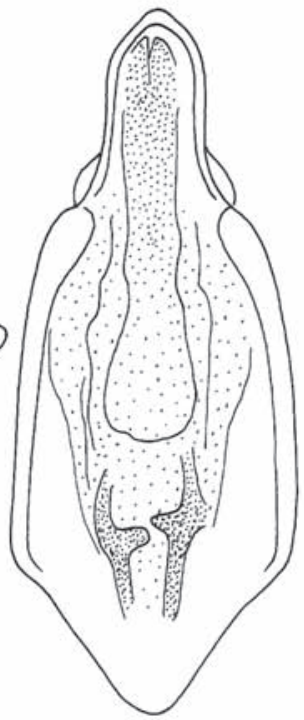

67

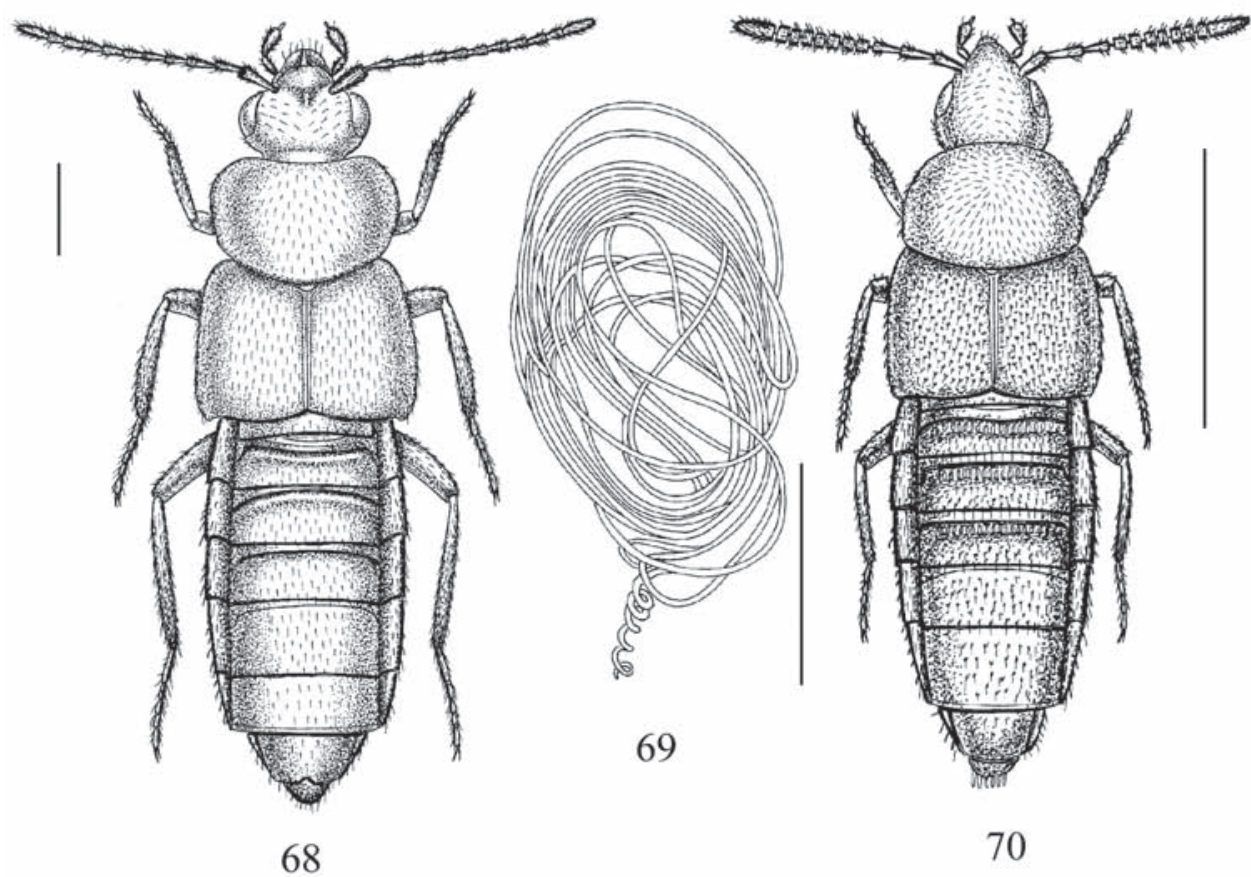

Figg. 65-70: Habitus, edeago in visione laterale e ventrale e spermateca. 65-67. Macrogerodonia unidens n. sp.; 68-69. Zyras (Diaulaconia) carinilabiatus n. sp.; 70. Tinotus densissimus Bernhauer, 1934. Habitus scala $1 \mathrm{~mm}$, altre scale $0,1 \mathrm{~mm}$. Habitus scala $1 \mathrm{~mm}$, altre scale $0,1 \mathrm{~mm}$. 

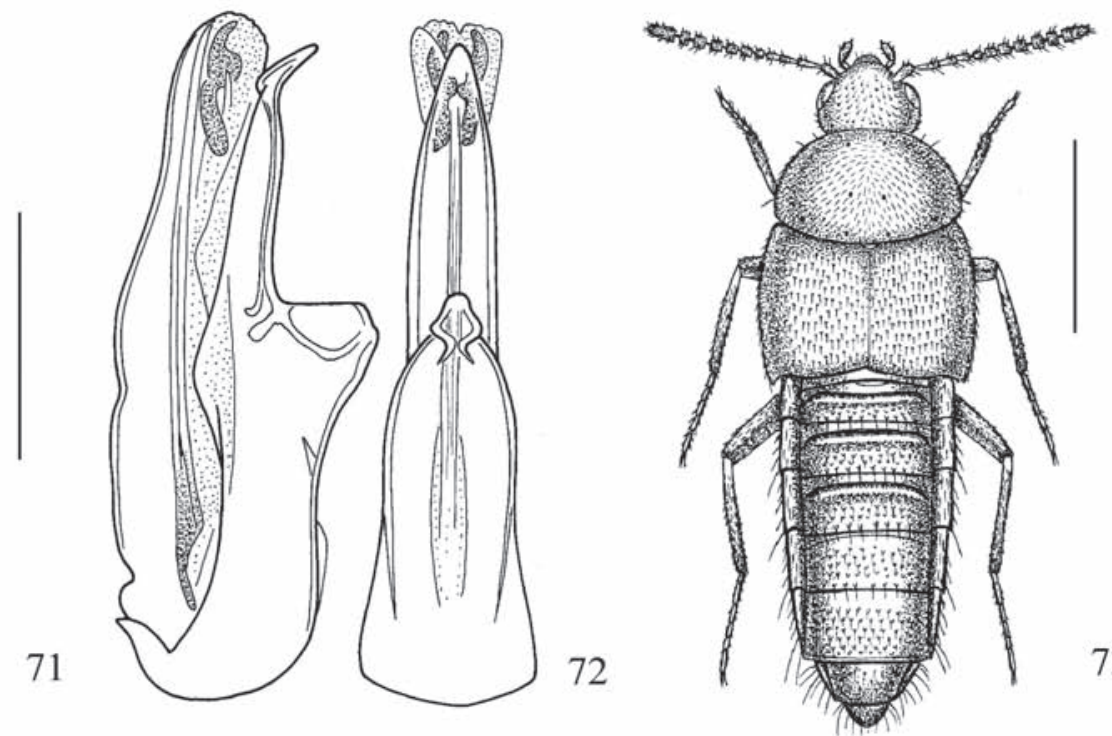

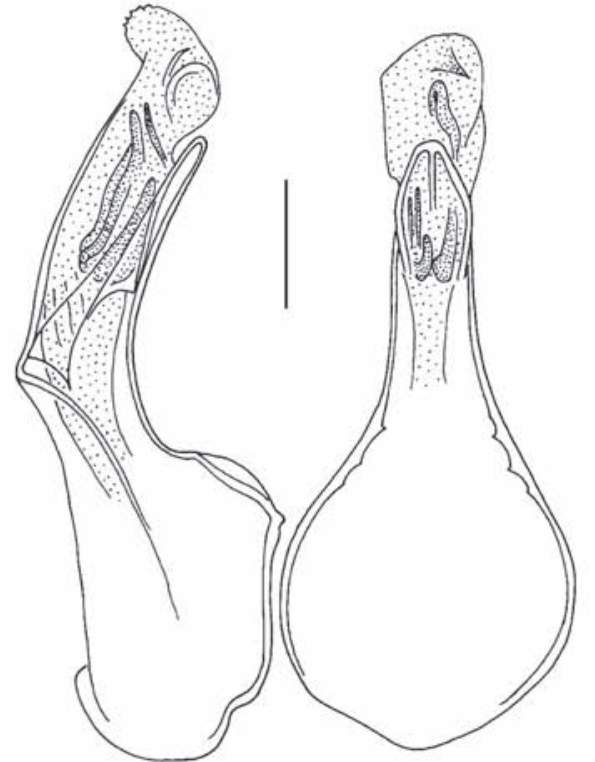

74

75

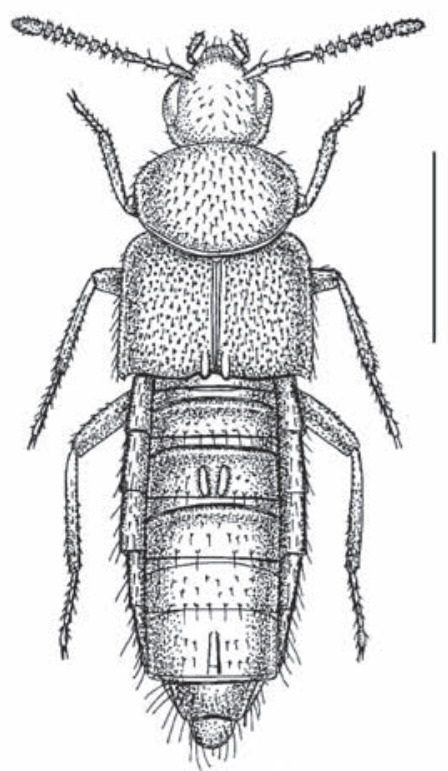

76

Figg. 71-76: Edeago in visione laterale e ventrale e habitus. 71-72. Tinotus densissimus Bernhauer, 1934; 73-75. Platandria crucis n. sp.; 76. Hoplandria diaghita n. sp. Habitus scala $1 \mathrm{~mm}$, altre scale $0,1 \mathrm{~mm}$. 

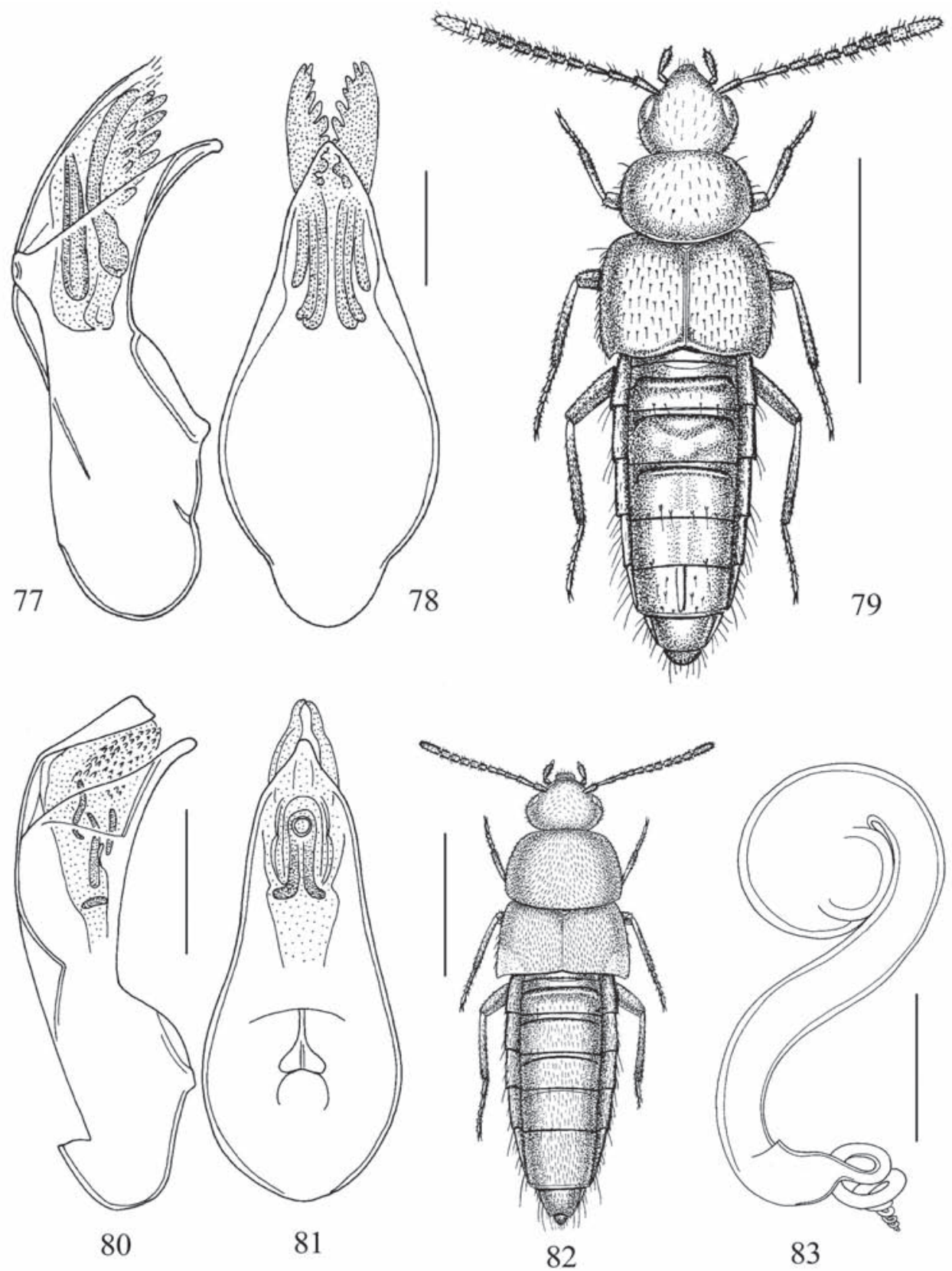

Figg. 77-83: Edeago in visione laterale e ventrale, habitus e spermateca. 77-78. Hoplandria diaghita n. sp.; 79-81. Hoplandria alboapicalis n. sp.; 82-83. Abrophaena franzi n. sp. Habitus scala $1 \mathrm{~mm}$, altre scale $0,1 \mathrm{~mm}$. 


\section{Oxypodini}

\section{Abrophaena franzi n. sp.}

(Figg. 82-83)

Materiale tipico: Holotypus $\uparrow$, S-Brasilien, Umg. Porto Alegre, b. Ponte de Guaiba, 1963, leg. H. Franz (NHMW).

\section{Descrizione:}

Lunghezza $3 \mathrm{~mm}$. Corpo lucidissimo e giallo-rossiccio con capo e uroterghi liberi quarto e quinto bruno-rossicci, elitre bruno-rossicce con angoli posteriori esterni gialli; antenne e zampe gialle. Granulosità finissima e saliente su tutto il corpo. Reticolazione assente su tutto il corpo. Uroterghi liberi con due solchi trasversi basali. Spermateca fig. 83 .

\section{Comparazioni:}

A motivo della forma della spermateca, la formula tarsale e la forma della ligula la nuova specie si pone tassonomicamente accanto ad A. boliviana PACE, 1983, della Bolivia, ma la nuova specie ha pronoto più ristretto in avanti che all'indietro (pronoto ristretto in avanti come all'indietro in boliviana) e il bulbo distale della spermateca molto sviluppato, molto più largo della porzione intermedia della stessa spermateca, mentre in boliviana esso è più stretto della porzione intermedia.

Etimologia: La nuova specie è dedicata alla memoria del suo raccoglitore, il prof. Herbert Franz, noto studioso della coleotterofauna del suolo.

\section{Meoticaops boliviensis n. sp.}

(Figg. 84-87)

Materiale tipico: Holotypus o $^{\star}$, Bolivien, Villa Montes, 22.XI.1930, leg. Dr. Eisentraut (MB). Paratypi: 3 우 옹 stessa provenienza (MB), (MRSNT).

\section{Descrizione:}

Lunghezza 1,7 mm. Corpo lievemente opaco e giallo-bruno con capo bruno-rossiccio e pronoto rossiccio; antenne giallo-rossicce con i due antennomeri basali giallo-rossicci; zampe gialle. Granulosità del capo fittissima, tanto da dare un aspetto scabro della superficie, quella del pronoto e delle elitre con granuli meno fini di quelli del capo, sempre fittissimi. Reticolazione assente su tutto il corpo. Solo il primo urotergo libero del maschio solcato alla base. Edeago figg. 85-86, spermateca fig. 87.

\section{Comparazioni:}

La nuova specie è attribuita al genere Meoticaops PACE, 1983, del Venezuela, oltre che per la ligula intera e a base larga, a motivo della forma della sua spermateca simile a quella di $M$. franzi PACE, 1983, del Venezuela. La nuova specie ha elitre più lunghe del pronoto, mentre in franzi sono più corte del pronoto; è priva, inoltre, di appendice distale del bulbo distale della spermateca, presente invece in franzi.

Etimologia: La nuova specie prende nome dalla Bolivia. 
Beitr. Ent. 59 (2009) 1

Apimela boliviana n. sp.

(Figg. 88-89)

Materiale tipico: Holotypus 우, Bolivien, Villa Montes, 17.XI.1930, leg. Dr. Eisentraut (MB).

\section{Descrizione:}

Lunghezza 1,9 mm. Corpo debolmente lucido e giallo-bruno con capo, elitre, tranne gli omeri, e quarto urotergo libero bruni; antenne brune con i due antennomeri basali gialli; zampe gialle. Granulosità dell'intero corpo fittissima, tanto da sembrare una reticolazione evidente. Spermateca fig. 89.

\section{Comparazioni:}

Per avere gli occhi molto sviluppati ed elitre più lunghe del pronoto, la nuova specie è molto differente da $A$. chilensis PACE, 1987, del Cile. La nuova specie è invece comparabile con $A$. errans PACE, 1990, del Brasile a motivo dell'habitus molto simile, ma gli occhi della nuova specie sono lunghi quanto le tempie (occhi più corti delle tempie in errans) e la spermateca è composta di una matassa stretta, mentre è molto larga in errans.

Etimologia: La nuova specie prende nome dalla Bolivia.

\section{Feluva colubrina (ERICHSON, 1839), comb. n.}

Phloeopora colubrina ERICHSON, 1839: 78

$10^{\star}$ e 1 ㅇ, Nova Teutonia, 1957, leg. Plaumann (MB) (serie tipica da me esaminata $\mathrm{N}^{\circ}$ 5345, $\mathrm{MB})$.

Distribuzione: Specie già nota del Brasile e Colombia.

Nota:

La specie tipo del genere Brachyglossa Fauvel, 1866 è B. varicolor Fauvel, 1866, del Cile. BlackWELDER (1952) ha scoperto che il nome Brachyglossa è pre-occupato da Brachyglossa Boisduval, 1828 e ha dato al genere il nuovo nome Feluva. Il mio esame della serie tipica di Feluva varicolor Fauvel, del Cile, e della serie tipica di Phloeopora colubrina Erichson, del Brasile, mi ha permesso di riconoscere con certezza che colubrina appartiene al genere Feluva. La ligula, infatti, è intera e larga nelle due specie e la spermateca è avvolta in spire in entrambe le specie.

\section{Feluva punctulata n. sp.}

(Figg. 90-93)

Materiale tipico: Holotypus $0^{\star}$, Columbia, Rio Cauca (MB).

Paratypus: 1 ㅇ, stessa provenienza (MRSNT).

\section{Descrizione:}

Lunghezza 2,7 mm. Corpo lucido e bruno con elitre e addome bruno-rossicci, quarto urotergo libero bruno; antenne bruno-rossicce con primo antennomero basale e base del secondo rossicci; zampe rossicce. Punteggiatura del capo assai forte, ma assente sulla fascia longitudinale mediana, quella del pronoto superficiale e quella delle elitre forte. Reticolazione del capo forte e molto trasversa, longitudinale sulla fascia longitudinale mediana, quella di pronoto ed elitre evidente e 


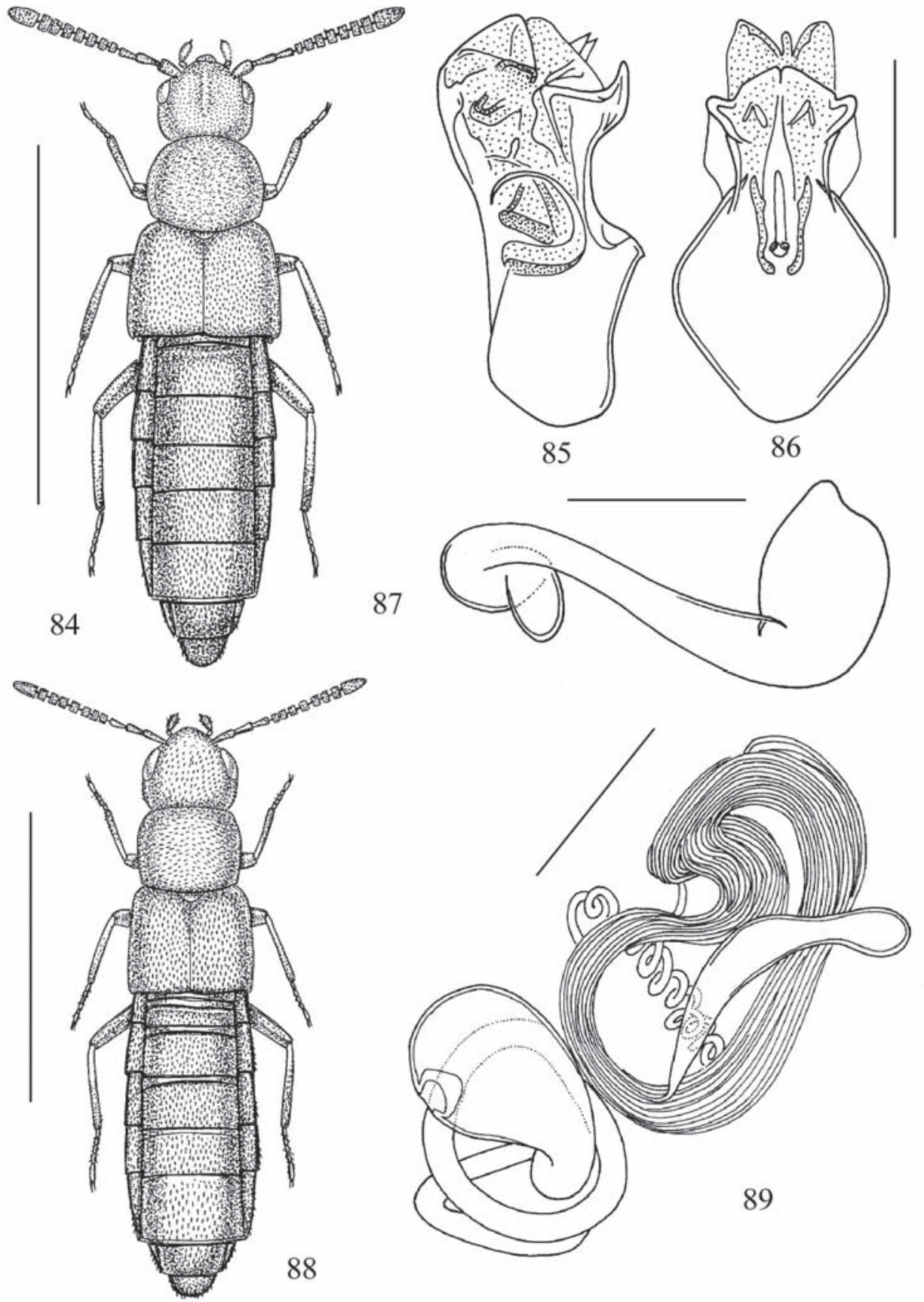

Figg. 84-89: 84-87. Meoticaops boliviensis n. sp.; 88-89. Apimela boliviana n. sp. Habitus scala $1 \mathrm{~mm}$, altre scale $0,1 \mathrm{~mm}$. Habitus scala $1 \mathrm{~mm}$, altre scale $0,1 \mathrm{~mm}$. 
fine, quella dell'addome molto trasversa e molto superficiale, solo sul quinto urotergo libero forte. Pronoto appiattito nel mezzo posteriormente. Uroterghi liberi con quattro solchi basali, nel fondo con punti forti radi. Edeago figg. 91-92, spermateca fig. 93.

\section{Comparazioni:}

Le specie finora note del genere Feluva Blackwelder, 1952, F. varicolor (Fauvel, 1866), del Cile, (di cui ho esaminato la serie tipica), F. colubrina (Erichson, 1839), del Brasile (di cui ho esaminato l'holotypus $\sigma^{\text {T }}$ ) e altre specie da me descritte, presentano capo ed elitre con punteggiatura da poco visibile a superficiale. La punteggiatura del capo e delle elitre della nuova specie, invece, è forte. La spermateca della nuova specie ha introflessione apicale del bulbo distale breve, mentre è profonda in F. guadalupensis PACE, 1987 e le altre specie sono prive di introflessione apicale del bulbo distale della spermateca.

Etimologia: La nuova specie prende nome di "punteggiata» a motivo della presenza di una forte punteggiatura del capo e delle elitre.

\section{Feluva minuta n. sp.}

(Figg. 94-96)

Materiale tipico: Holotypus ơ ${ }^{\star}$, Colombia, Valle del Cauca, a nordest della città di Cali (MB).

Paratypi: 1 o e 1 \& (senza spermateca), stessa provenienza (MRSNT).

\section{Descrizione:}

Lunghezza 2,3 $\mathrm{mm}$. Corpo opaco e bruno-rossiccio; antenne rossicce; zampe giallo-rossicce. Punteggiatura del capo assente. Granulosità del pronoto e delle elitre poco visibile. Reticolazione dell'avancorpo forte, quella dell'addome molto trasversa e assai superficiale. Pronoto con un debole solco mediano anteriore. Uroterghi liberi con tre solchi trasversi basali, punteggiati nel fondo. Sesto urotergo libero del maschio con granuli salienti sulla metà basale, assenti sulla metà posteriore. Edeago figg. 95-96.

\section{Comparazioni:}

La nuova specie è affine a $F$ guadalupensis PACE, 1987, per la forma dell'edeago. Se ne distingue per avere il quarto antennomero più lungo che largo (trasverso in guadalupensis) e per l'edeago rettilineo all'apice distale, in visione laterale, e non sinuato come in guadalupensis.

Etimologia: La nuova specie prende nome di «piccola» per la breve sua taglia corporea.

\section{Idiostiba venezuelensis $\mathrm{n} . \mathrm{sp}$.}

(Figg. 97-99)

Materiale tipico: Holotypus ơ, Venezuela, Colonia Tovar, b. Maracay, 2000 m, leg. H. Franz (MHMW).

\section{Descrizione:}

Lunghezza 3,3 $\mathrm{mm}$. Corpo lucidissimo e bruno-rossiccio, con capo nero, pronoto e uroterghi liberi terzo, quarto e quinto bruni; antenne brune con i due antennomeri basali giallo-rossicci; zampe giallo-rossicce. Punteggiatura del capo fine, evidente e rada, quella delle elitre meno fine, altrettanto evidente. Granulosità del pronoto ben visibile. Reticolazione dell'avancorpo assente, quella dell'addome molto trasversa e molto superficiale. Edeago figg. 98-99. 

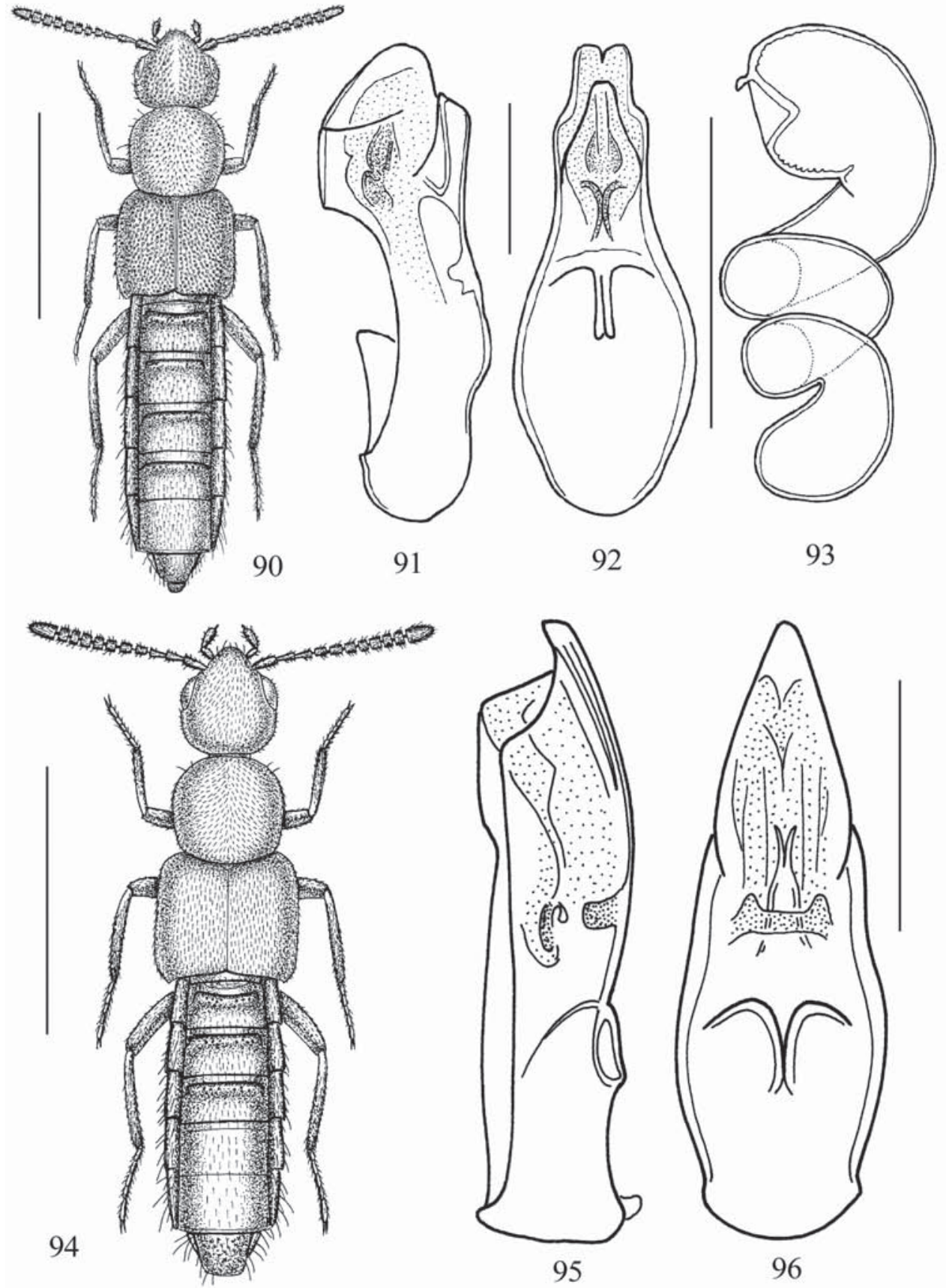

Figg. 90-96: Habitus, edeago in visione laterale e ventrale e spermateca. 90-93. Feluva punctulata n. sp.; 94-96. Feluva minuta n. sp. Habitus scala $1 \mathrm{~mm}$, altre scale $0,1 \mathrm{~mm}$. 


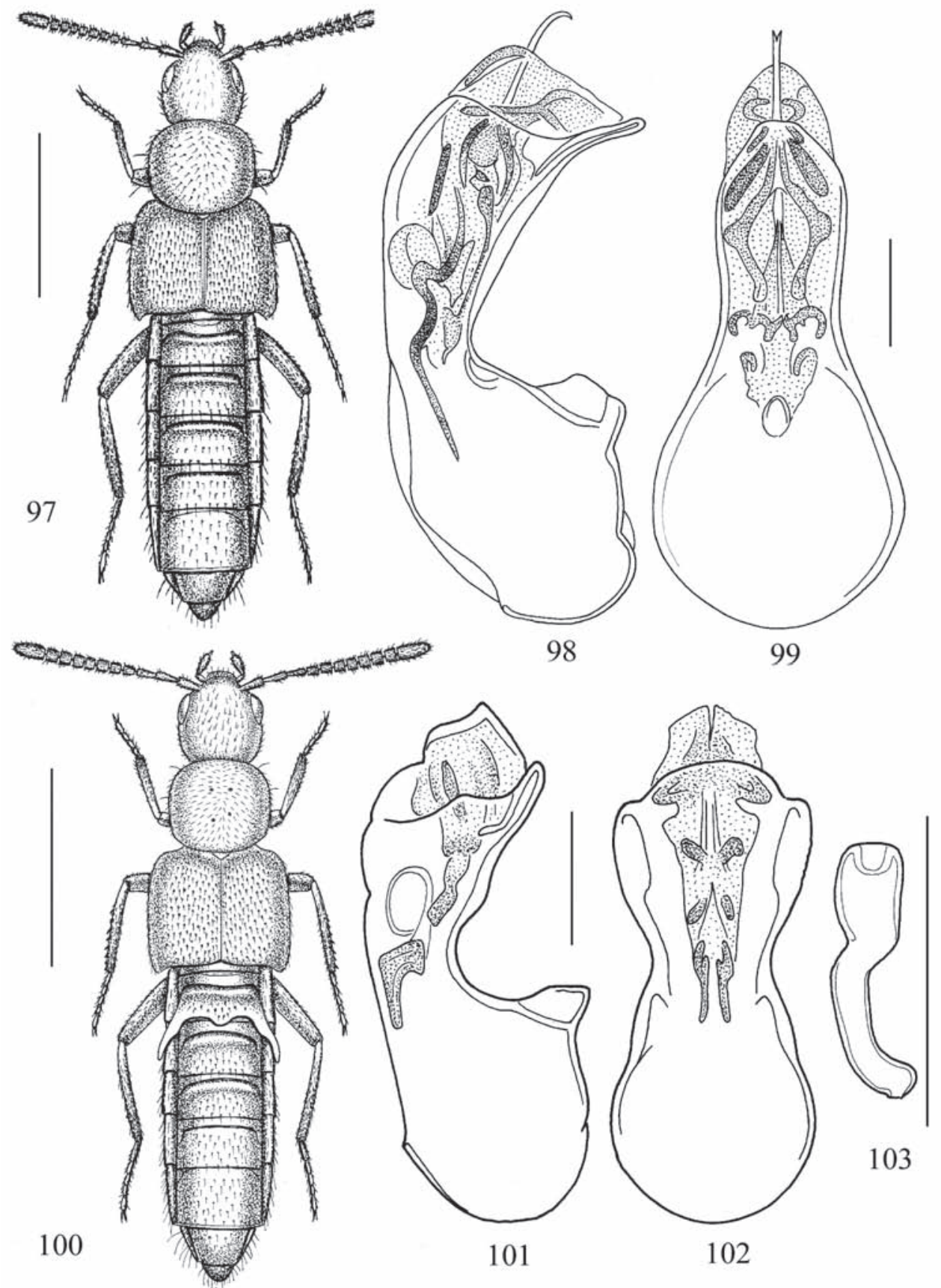

Figg. 97-103: Habitus, edeago in visione laterale e ventrale e spermateca. 97-99. Idiostiba venezuelensis n. sp.; 100-103. Diacanthochara brasiliensis n. sp. Habitus scala $1 \mathrm{~mm}$, altre scale $0,1 \mathrm{~mm}$. 


\section{Comparazioni:}

L'habitus della nuova specie è simile a quello di I. missionum (BERnHAUER, 1934) comb. n., dell'Argentina («olim» Atheta (Microdota) missionum Bernhauer, 1934: 503) (holotypus + da me esaminato). Se ne distingue per avere il quarto antennomero lungo quanto largo (fortemente trasverso e minuscolo rispetto al seguente in missionum) e per la pubescenza dell'addome poco fitta e non fittissima come in missionum.

Etimologia: La nuova specie prende nome dal Venezuela.

\section{Diacanthochara brasiliensis n. sp.}

(Figg. 100-103)

Materiale tipico: Holotypus đૈ, Brasilien, Nova Teutonia, VI.1960, 300-500 m, leg. Plaumann (MB).

Paratypi: 3 우 오 , stessa provenienza (MB), (MRSNT).

\section{Descrizione:}

Lunghezza 2,8 $\mathrm{mm}$. Corpo lucidissimo e rossiccio con elitre bruno-rossicce; antenne brune con i tre antennomeri basali giallo-rossicci; zampe rossicce. Punteggiatura del capo e del pronoto superficiale, quella delle elitre e dell'addome evidente. Reticolazione del corpo assente, tranne che sulle elitre su cui è superficiale. Quattro punti forti discali sul pronoto. Primo urotergo libero del maschio con due prolungamenti laterali e un lobo mediano al margine posteriore. Edeago figg. 101-102, spermateca fig. 103.

\section{Comparazioni:}

La nuova specie è simile a $D$. venezuelana PACE, 1983, del Venezuela, per la forma dell'edeago, ma questo nella nuova specie è molto dilatato nella porzione apicale distale, in visione ventrale, mentre è stretto in venezuelana. Il quarto antennomero, inoltre, è lungo quanto largo nella nuova specie, trasverso in venezuelana. La spermateca è semplice nella nuova specie e avvolta in numerose spire in venezuelana.

Etimologia: La nuova specie prende nome dal Brasile.

\section{Acrocyusa forticornis n. sp.}

(Figg. 104-105)

Materiale tipico: Holotypus ㅇ, Brasilien, Nova Teutonia, VII.1955-XI.1955, 300-500 m, leg. Plaumann (MB).

Paratypi: 3 우 오 , stessa provenienza (MB), (MRSNT).

\section{Descrizione:}

Lunghezza $3 \mathrm{~mm}$. Corpo lucidissimo e rossiccio, con quarto urotergo libero bruno-rossiccio; antenne bruno-rossicce con i due antennomeri basali giallo-rossicci; zampe rossicce. Punteggiatura del capo evidente e assente sulla fascia longitudinale mediana, quella del pronoto fine e molto superficiale. Granulosità delle elitre saliente, quella dell'addome fine. Reticolazione assente su tutto il corpo. Quattro punti forti sul disco del pronoto. Solchi trasversi basali punteggiati. Spermateca fig. 105. 
Nota:

Acrocyusa Bernhauer, 1930, è stato descritto come sottogenere di Ocyusa Kraatz, 1856. In questa sede è considerato genere a sé stante per le antenne molto clavate.

\section{Comparazioni:}

A motivo delle antenne fortemente clavate, la nuova specie è simile ad A. grandicornis BERNHAUER, 1930, del Congo. Se ne distingue per avere il corpo rossiccio con quarto urotergo libero bruno-rossiccio (nero e nero-bruno in grandicornis), per l'undicesimo antennomero brunorossiccio e non giallo-rossiccio come in grandicornis, per gli occhi molto più corti delle tempie (occhi lunghi quanto le tempie in grandicornis) e per l'undicesimo antennomero lungo quanto i quattro precedenti riuniti (il medesimo poco più lungo dei due antennomeri precedenti riuniti in grandicornis).

Etimologia: Il nome della nuova specie significa «antenne forti» a motivo delle sue robuste antenne.

\section{Neocalodera iguazuensis $\mathrm{n} . \mathrm{sp}$.}

(Figg. 106-108)

Materiale tipico: Holotypus ơ, Brasilien, Nova Teutonia, VII.1959, leg. Plaumann (MB).

\section{Descrizione:}

Lunghezza $4 \mathrm{~mm}$. Corpo lucido e bruno con margine posteriore degli uroterghi liberi rossiccio; antenne brune con i due antennomeri basali e base del terzo giallo-rossicci; zampe rossicce. Punteggiatura del capo e delle elitre profonda e forte. Granulosità del pronoto poco saliente, quella dell'addome molto sparsa sugli uroterghi liberi terzo e quarto. Reticolazione assente su tutto il corpo. Fossetta trasversa basale del pronoto. Solchi trasversi basali degli uroterghi liberi punteggiati. Sesto urotergo libero del maschio crenellato al margine posteriore, tra i due lobi laterali. Edeago figg. 107-108.

\section{Comparazioni:}

La nuova specie per la forma dell'edeago è tassonomicamente in posizione intermedia tra $N$. arcuata (Fauvel, 1866) (holotypus ơ da me esaminato) e N. magellanensis PACE, 1987, entrambe del Cile. Gli occhi della nuova specie sono lunghi quanto le tempie, mentre nelle due specie a confronto sono più corti delle tempie. Il pronoto della nuova specie presenta una fossetta mediana trasversa posteriore come in arcuata, ma questa presenta punteggiatura del capo superficiale e non forte come nella nuova specie. L'edeago della nuova specie è profondamente e largamente arcuato al lato ventrale, mentre quello di arcuata è ampiamente, ma non profondamente arcuato, quello di magellanensis è profondamente, ma strettamente arcuato.

Etimologia: La nuova specie prende nome dalle famose cascate dell'Iguazù.

\section{Neocalodera gratella n. sp.}

(Figg. 109-110)

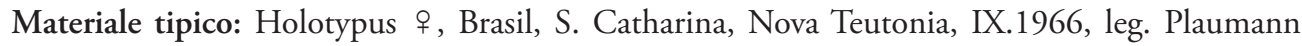
(MB). 


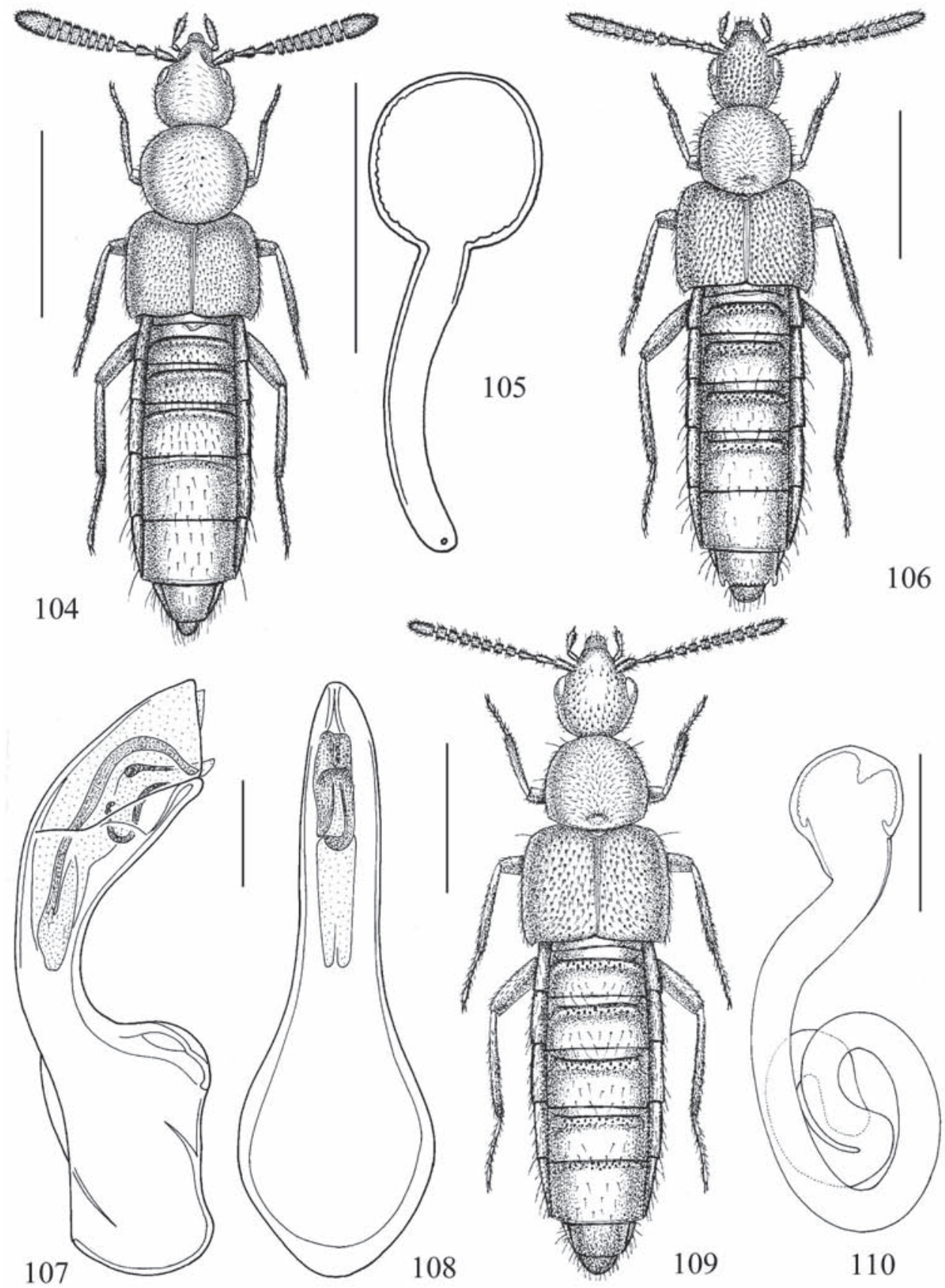

Figg. 104-110: Habitus, spermateca e edeago in visione laterale e ventrale. 104-105. Acrocyusa forticornis n. sp.; 106-108. Neocalodera iguazuensis n. sp.; 109-110. Neocalodera gratella n. sp. Habitus scala $1 \mathrm{~mm}$, altre scale $0,1 \mathrm{~mm}$. 


\section{Descrizione:}

Lunghezza $4 \mathrm{~mm}$. Corpo lucidissimo e bruno, con margine posteriore degli uroterghi liberi rossiccio; antenne brune con antennomero basale rossiccio e secondo bruno-rossiccio; zampe bruno-rossicce con tarsi giallo-rossicci. Punteggiatura del capo forte, assente sul disco, quella del pronoto superficiale, quella delle elitre evidente. Reticolazione assente su tutto il corpo. Pronoto con fossetta trasversa mediana basale. Quattro solchi trasversi basali degli uroterghi liberi con fondo punteggiato. Spermateca fig. 110.

\section{Comparazioni:}

La nuova specie presenta habitus simile a quello di $N$. arcuata (FAuvel, 1866) (holotypus ơ da me esaminato), del Cile. Se ne distingue per la punteggiatura del capo forte e assente sul disco e non molto superficiale e fittissima come in arcuata, per le antenne brune con i due antennomeri basali bruno-rossicci e non interamente rossicce come in arcuata e per la punteggiatura delle elitre uniformemente distribuita ed evidente e non addensata alla base e superficiale sulla metà posteriore come in arcuata.

Etimologia: Il nome della nuova specie significa «un po’ gradita».

\section{Spanioda splendens (PACE, 1987)}

Spanioda splendens PACE, 1987: 496

1 ㅇ, Chile (MB).

Distribuzione: Specie già nota del Cile.

\section{Spanioda brasiliana n. sp.}

(Figg. 111-113)

Materiale tipico: Holotypus ơ, S. Brasilien, Theresopolis, XU.1904, leg. Michaelis (MB).

\section{Descrizione:}

Lunghezza $4 \mathrm{~mm}$. Corpo lucidissimo e nero-bruno con pigidio rossiccio; antenne giallorossicce con i tre antennomeri basali gialli; zampe giallo-rossicce. Punteggiatura del capo e delle elitre molto superficiale, quella del pronoto evanescente e infittita sulla fascia longitudinale mediana. Reticolazione assente su tutto il corpo. Pronoto con lieve depressione mediana posteriore. Solchi trasversi basali dei quattro uroterghi liberi basali con punteggiatura forte. Edeago figg. 112-113.

\section{Comparazioni:}

La nuova specie presenta pronoto notevolmente sinuato ai lati. Questo carattere, anche se attenuato, si osserva anche in S. pectoralis (Solier, 1849), del Cile (serie tipica da me esaminata). Oltre ad avere il pronoto molto sinuato ai lati, la nuova specie presenta il quarto antennomero assai più lungo che largo (poco più lungo che largo in pectoralis) e l'edeago è fortemente e bruscamente dilatato nella porzione distale, in visione ventrale, e non a lati quasi paralleli come in pectoralis.

Etimologia: La nuova specie prende nome dal Brasile. 


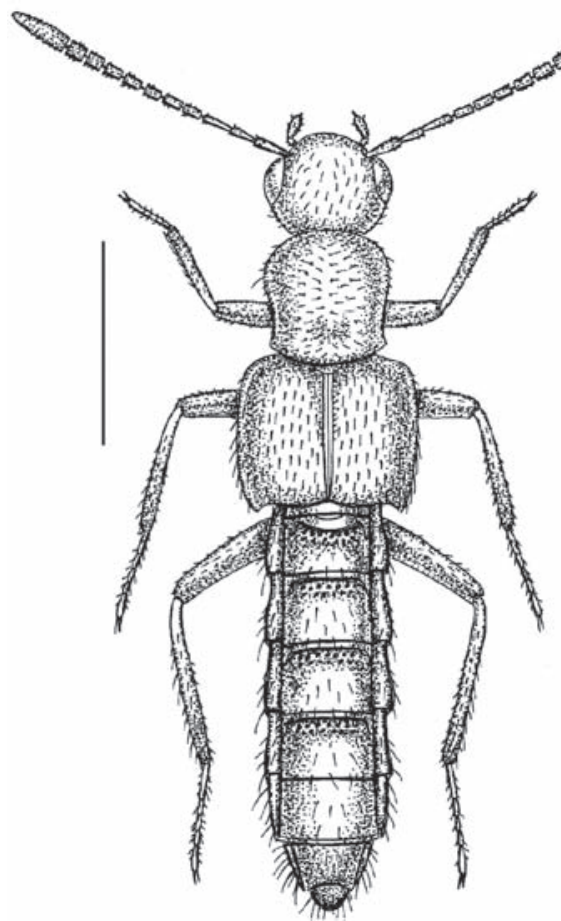

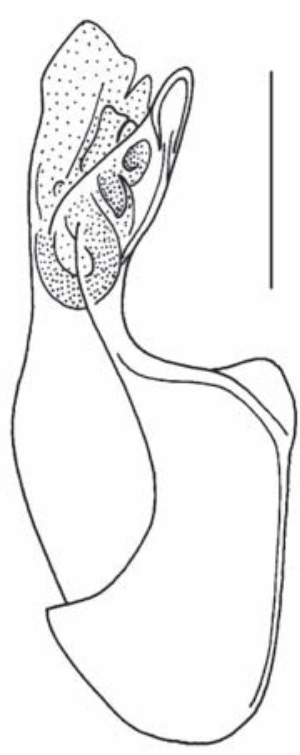

112

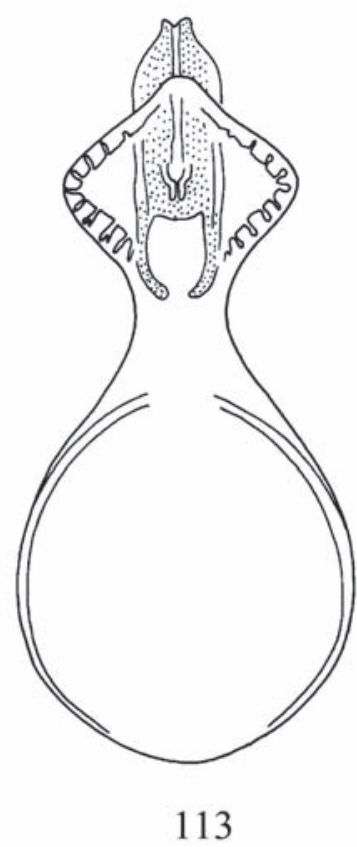

111

Figg. 111-113: Habitus e edeago in visione laterale e ventrale. 111-113. Spanioda brasiliana n. sp. Habitus scala $1 \mathrm{~mm}$, altra scala $0,1 \mathrm{~mm}$.

\section{Hoplandriini}

\section{Tinotus densissimus BERNHAUER, 1934}

Tinotus densissimus Bernhauer, 1934: 512

1 đ̆$^{\star}$, Rio Jan., Sta. Cruz, leg. Dr. Hensel (MB), figg. 70-72.

Distribuzione: Specie già nota di Buenos Aires. Nuova per il Brasile.

\section{Platandria crucis n. sp.}

(Figg. 73-75)

Materiale tipico: Holotypus ơ, Bolivien, Umg. Sta. Cruz, leg. H. Franz (NHMW).

\section{Descrizione:}

Lunghezza 3,1 mm. Corpo lucido e nero-bruno con elitre brune con fascia obliqua brunorossiccia dall'omero all'angolo posteriore interno; antenne bruno-rossicce con i sei antennomeri basali giallo-rossicci; zampe bruno-rossicce con ginocchia e tarsi nero-bruni. Granulosità del capo 
e delle elitre saliente. Punteggiatura del pronoto ombelicata evidente. Reticolazione del capo e dell'addome superficiale (tranne che sul quinto urotergo libero su cui è evidente), quella del pronoto molto evanescente e quella delle elitre evidente. Tre punti forti allineati longitudinalmente a ciascun lato della fascia longitudinale mediana del pronoto. Solchi trasversi basali degli uroterghi liberi basali senza punteggiatura. Edeago figg. 74-75.

\section{Comparazioni:}

La nuova specie presenta edeago simile a quello di P. neotropica BIERING, 1939, della Costa Rica, (serie tipica esaminata da GÉNIER \& KLIMASZEwsKi, 1986), ma la porzione distale, in visione ventrale, è molto più stretto di quello di neotropica e l'armatura genitale interna dell'edeago è molto più sviluppata nella nuova specie che in neotropica.

Etimologia: La nuova specie prende nome dalla città di Santa Cruz in Bolivia.

\section{Hoplandria diaghita n. sp.}

(Figg. 76-78)

Materiale tipico: Holotypus ơ, Argentinien, Salta, 1200 m, II.1905, leg. J. Steinbach (MB).

\section{Descrizione:}

Lunghezza 3,5 mm. Corpo lucido e giallo-rossiccio con capo rossiccio; antenne rossicce con i tre antennomeri basali giallo-rossicci; zampe giallo-rossicce. Punteggiatura del capo evidente, quella del pronoto e delle elitre forte. Reticolazione del corpo assente. Ciascun angolo interno posteriore delle elitre con una carena saliente. Sono presenti due carene mediane posteriori sul secondo urotergo libero del maschio e una carena mediana posteriore sul quinto urotergo libero del maschio. Edeago figg. 77-78.

\section{Comparazioni:}

La nuova specie può essere tassonomicamente vicina a H. umbrina KRAATZ, 1857, della Colombia (serie tipica da me esaminata) a motivo della presenza di alcuni caratteri delle elitre e dell'edeago. Se ne distingue per il quarto antennomero trasverso (più lungo che largo in umbrina), per il dente dell'angolo posteriore interno delle elitre del maschio forte (esile in umbrina), per la presenza di due carene mediane sul secondo urotergo libero del maschio (assenti in umbrina) e per l'armatura genitale interna dell'edeago composta da due forti e lunghi pezzi copulatori e non otto corti come in umbrina.

Etimologia: La nuova specie prende nome dai Diaghiti, antica popolazione della valle di Tucuman.

\section{Hoplandria alboapicalis n. sp.}

(Figg. 79-81)

Materiale tipico: Holotypus ${ }^{\star}$, Peru, Umg. Tarapoto, leg. H. Franz (MHMW).

\section{Descrizione:}

Lunghezza 2,8 mm. Corpo lucido e giallo-rossiccio, elitre bruno-rossicce; antenne brune con i tre antennomeri basali e base del quarto giallo-rossicci e decimo e undicesimo giallo-chiari; zampe 
giallo-rossicce con femori gialli. Punteggiatura del capo e del pronoto poco evidente. Granulosità delle elitre saliente e fine. Angolo posteriore interno delle elitre sollevato. Bozza mediana posteriore sul secondo urotergo libero del maschio, terzo con spigolo longitudinale mediano, quinto con carena mediana saliente. Edeago figg. 80-81.

\section{Comparazioni:}

L'edeago della nuova specie è simile a quello di $H$. fassli Bernhauer, 1920, della Colombia (serie tipica da me esaminata). I due antennomeri apicali della nuova specie sono giallo-chiari, mentre in fassli il decimo antennomero è bruno e l'undicesimo rossiccio. L'angolo posteriore interno delle elitre del maschio è saliente nella nuova specie e ornato di una lunga spina in fassli. L'armatura genitale interna dell'edeago della nuova specie è esile rispetto quella di fassli che è robusta.

Etimologia: La nuova specie prende nome di «apice bianco». L’apice bianco è quello delle antenne.

\section{Aleocharini}

\section{Ctenopeuca heynei BERnhAUER, 1915}

Ctenopeuca heynei Bernhauer, 1915: 300

$2 \sigma^{\top} o^{\star}$, Peru Chanchamayo, XI (MB).

Distribuzione: Specie già nota del Perù. Serie tipica da me esaminata.

\section{Aleochara (Aleochara) lustrica SAY, 1836}

Aleochara lustrica SAY, 1836: 468

Aleochara (Aleochara) lustrica; KLIMAszewski, 1984: 72

12 es., Brasilien, Bahia (MB), (MRSNT).

Distribuzione: Specie nota degli Stati Uniti d'America e Messico. Nuova per il Brasile.

\section{Aleochara (Aleochara) parvicollis BERNHAUER, 1904}

Aleochara (s. str.) parvicollis BernHAuer, 1904: 241

31 es., Peru, Dep. Cuzco, Cosnipata Eben, Cajon Berglad, 1900, leg. Garlepp S.V. (MB), (MRSNT).

Distribuzione: Specie finora nota solo del Perù.

\section{Aleochara (Aleochara) atra (SOLIER, 1849)}

Mecorhopalus ater Solier, 1849: 348

Aleochara (s. str.) atra; BeRnHAUER \& SCHEERPELTZ, 1926: 776

2 우 우, Chile, Osorno; 5 es., Chile, Santiago, Puelma (MB).

Distribuzione: Specie finora nota solo del Cile. 


\section{Aleochara (Xenochara) fumata GrAvENHORST, 1802}

Aleochara fumata GravenHoRst, 1802: 96

Aleochara (Xenochara) fumata; KuIMASZEwsKi, 1984: 51

1 ơ e 1 ㅇ, Nova Teutonia, leg. Plaumann; 5 es., Argentine, prov. Salta, leg. Steinbach; 4 es., Comitan (MB).

Distribuzione: Specie nota della regione paleartica e del Nordamerica. Nuova per Brasile e Argentina.

\section{Aleochara (Xenochara) weiseri BERNHAUER, 1921}

Aleochara weiseri BernhaUer 1921: 178

Aleochara (Xenochara) weiseri; KLIMAszewski \& Maus, 1999

15 es., Paraguay, leg. Fieber (MB), (MRSNT); 2 es., Nova Teutonia, leg. Plaumann (MB).

Distribuzione: Specie nota dell'Argentina. Nuova per il Paraguay.

\section{Aleochara (Xenochara) lacertina SHARP, 1883}

Aleochara lacertina SHARP 1883: 148

Aleochara (Xenochara) lacertina; KLIMASZEWski, 1984: 39

$10^{\star}$, Lima $(\mathrm{MB})$.

Distribuzione: Specie nota del Nordamerica e del Messico. Nuova per il Perù.

\section{Aleochara (Coprochara) notula ERICHSON, 1839}

Aleochara notula ERICHSON, 1839: 167

Aleochara (Coprochara) notula; KLIMASZEWsKi, 1984: 20

$1 \sigma^{\star}$, Chile, Santiago, Puelma.

Distribuzione: Specie già nota degli Stati Uniti d'America, Messico, Venezuela e Cile. Serie tipica di 2 우 da me esaminata.

\section{Aleochara (Coprochara) bimaculata GRAVENHORST, 1802}

Aleochara bimaculata Gravenhorst, 1802: 187

Aleochara (Coprochara) bimaculata; KLIMASZEwsKi, 1984: 17

6 es., Bogotà (MB); 6 es., Kuba, Varadero, 2.XII.1980, leg. Ü. Golbner (MB), (MRSNT); 10 es., Mato Grosso, Rohde (MB), (MRSNT).

Distribuzione: Specie già nota degli Stati Uniti d'America, Canada, Messico, Colombia, Argentina. Nuova per Cuba. 


\section{Ringraziamenti}

Per avermi affidato in studio il materiale oggetto del presente lavoro rivolgo i miei più cordiali ringraziamenti al Dr. Manfred Uhlig del Museo Zoologico dell'Università Humboldt di Berlino e al Dr. Lothar Zerche del DEI di Müncheberg. Per il prestito di tipi ringrazio il Dr. A. F. Newton jr., del Field Museum of Natural History di Chicago e ancora il Dr. Manfred Uhlig e il Dr. Lothar Zerche.

\section{Bibliografia}

Bernhauer, M. 1904: Neue exotische Staphyliniden. - Stettiner Entomologische Zeitung 65: 239-242.

Bernhauer, M. 1915: Zur Staphyliniden-Fauna von Südamerika - Stettiner Entomologische Zeitung 76: 291-301.

Bernhauer, M. 1920a: Neue Aleocharini aus Südamerika. - Archiv für Naturgeschichte 86: 141-170.

Bernhauer, M. 1920b: Zur Kenntnis der Staphyliniden-Gattung Zyras Steph. XVII. Beitrag zur Staphylinidenfauna Afrikas. - Archiv für Naturgeschichte 92: 19-75.

Bernhauer, M. 1921: Neue Staphyliniden aus Südamerica, besonders aus Argentinien. - Wiener Entomologische Zeitung 38: 101-179.

Bernhauer, M. 1930: Neue Kurzflügler aus der Subfamilie Aleocharinae vom belgischen Kongostaate. 22. Beitrag zur afrikanischen Staphylinidenfauna. - Folia Zoologica et Hydrobiologica 2: 180-209.

Bernhauer, M. 1934: Neue Staphyliniden aus Argentiniens. - Revista de Entomologia do Rio de Janeiro 3: 501-517.

Bernhauer, M. 1940: Neue Staphyliniden aus Costa Rica. - Arbeiten über morphologische und taxonomische Entomologie aus Berlin-Dahlem 7: 158-161.

Bernhauer, M. 1941: Staphylinidae (Col.). - In: Beiträge zur Fauna Perous 1: 277-293.

Bernhauer, M. \& Scheerpeltz, O. 1926: Coleopterorum Catalogus, pars 82, Staphylinidae VI: 499-988, Berlin.

Biering, A. 1939: Neue neotropische Staphylinidae. - Arbeiten über morphologische und taxonomische Entomologie aus Berlin-Dahlem 6: 24-31.

BlaCKWELDER, R. E. 1952: The generic Names of the Beetle Family Staphylinidae. - Smithsonian Institution, Bulletin 200: 483 pp., Washington.

Erichson, W. K. 1839: Genera et species Staphylinorum Insectorum Coleopterorum familiae. 1: 1-400. - Berlin: F. H. Morin.

Fauvel, A. 1866: Faune de Chili. Insectes Coléoptères Staphylinides. - Bulletin de la Société Linnéenne de Normandie, 10: 250-353.

Fauvel, A. 1905: Staphylinidae exotiques nouveaux (3e partie). - Revue d'Entomologie (Caen) 24: 113-147.

Génier, F. \& Klimaszewsi, J. 1986: Review of the types of the genus Platandria Casey with a key to the species (Coleoptera: Staphylinidae: Aleocharinae. - The Coleopterists Bulletin 40: 201-216.

Gravenhorst, J. L. C. 1802: Coleoptera microptera Brunsvicensia nec non exoticorum quotquot exstant in collectionibus entomologorum Brunsvicensium in genera, familias et species distribuit. Brunsuigae: Carolus Reichard, LXIV + 206 pp.

Klimaszewski, J. 1984: A revision of the genus Aleochara Gravenhorst of America north of Mexico (Coleoptera: Staphylinidae, Aleocharinae). - Memoirs of the Entomological Society of Canada 129: $211 \mathrm{pp}$.

Kuimaszewski, J. \& Maus, C. 1999: Review of Bernhauer's Types of Aleochara from South America (Coleoptera: Staphylinidae: Aleocharinae). - Zoological Studies 38 (2): 207-221.

KraAtZ, G. 1856: Naturgeschichte der Insecten Deutschlands, Abtheilung I, Coleoptera, 2: 1-376. Nicolai, Berlin. 
KraAtz, G. 1857: Genera Aleocharinorum Illustrata. - Linnaea Entomologica 11: 1-43.

Lynch-Arribalzaga, F. 1884: Los Estafilinos de Buenos Aires. - Boletin de la Academia Nacional de Ciencias, Córdoba 7: 6-392.

PACE, R. 1983a. Nuove Aleocharinae brasiliane e boliviane del Museo Zoologico dell'Università Humboldt di Berlino (Coleoptera Staphylindae). - Giornale italiano di Entomologia 1: 295-316.

PACE, R. 1983b: Aleocharinae orofile del Venezuela raccolte dal Prof. Franz. II. Callicerini Athetae, Falagriini \& Oxypodini (Coleoptera Staphylinidae). - Bollettino della Società entomologica italiana, 115: $142-151$.

PACE, R. 1987a: Aleocharinae del Cile (Coleoptera, Staphylinidae). - Redia 70: 459-522.

PACE, R. 1987b: Aleocharinae dell'isola della Guadalupa (Antille) (Coleoptera: Staphylinidae). - Folia Entomologica Hungarica 48: 187-205.

PACE, R. 1990: Nuovi Falagriini, Hoplandriini ed Aleocharini della Regione Neotropica (Coleoptera, Staphylinidae) (LXXX Contributo alla conoscenza delle Aleocharinae). - Giornale italiano d'Entomologia 5: 157-180.

PACE, R. 1997: Aleocharinae della Colombia e dell'Ecuador. Parte III (conclusione). (Coleoptera, Staphylinidae). - Revue suisse de Zoologie 104: 17-48.

Sharp, D. 1883: Biologia Centrali-Americana: Insecta, Coleoptera. 1 (pt. 2). - Taylor and Francis, London: pp. 145-312.

Solier, A. J. J. 1849: Coleoptera. - In: GAY, Historia Fisica y Politica de Chile: Insecta, vol. 4: 105-508, pubblicazione privata, Paris.

Solsky, S. M. 1875: Staphylinides recueillis par M M. C. Jelsky et le Baron De Nolcken dans le Pérou et la Nouvelle Grenade. - Horae Societatis Entomologicae Rossicae 11: 1-24.

\section{Indirizzo dell'autore:}

ROBErto PACE

Via Vittorio Veneto, 13

37032 Monteforte d'Alpone (Verona)

Italia

e-mail: pace.ent@tiscali.it

\section{Subject editor:}

Dr. L. ZerChe 